\title{
A Surrogate Based Approach to Reduced-Order Dynamic Stall Modeling
}

\author{
Bryan Glaz, Li Liu ${ }^{\dagger}$ and Peretz P. Friedmann ${ }^{\ddagger}$ \\ The University of Michigan, Ann Arbor, MI, 48109, USA \\ and \\ Jeremy Bain ${ }^{\S}$ and Lakshmi N. Sankarף \\ Georgia Institute of Technology, Atlanta, GA, 30332, USA
}

\begin{abstract}
The surrogate-based recurrence framework (SBRF) approach to reduced-order nonlinear unsteady aerodynamic modeling associated with pitching/plunging airfoils subject to fixed or time-varying freestream Mach numbers is described. Using full-order solutions generated by the OVERFLOW CFD code, the SBRF reduced-order modeling approach is shown to effectively mimic full-order solutions of unsteady lift, moment, and drag under dynamic stall conditions, but at a fraction of the computational cost. In addition to accounting for realistic helicopter rotor blade dynamics, it is shown that the SBRF can model advancing rotor blade stall due to shock induced separation, as well as retreating blade stall associated with excessive angles of attack. Therefore, the SBRF reduced-order modeling approach is ideally suited for a variety of aeroelasticity and active/passive design optimization studies that require high fidelity aerodynamic response solutions with minimal computational expense.
\end{abstract}

\section{Nomenclature}

\begin{tabular}{|c|c|}
\hline$a$ & Speed of sound \\
\hline$b$ & Airfoil semi-chord \\
\hline$C_{l}, C_{m}, C_{d}$ & Airfoil lift, moment, and drag coefficients \\
\hline$E^{(j)}$ & Error for the $j^{\text {th }}$ test case \\
\hline$f_{x}$ & Vector of basis functions associated with assumed polynomials in kriging \\
\hline $\mathbf{F}$ & Matrix of basis functions associated with assumed polynomials in kriging \\
\hline$G_{q}, G_{y}$ & State transition function and state-to-output mapping function respectively \\
\hline$h$ & Airfoil plunge degree of freedom \\
\hline $\bar{h}$ & Oscillatory plunge amplitude \\
\hline$k_{1}$ & Reduced frequency corresponding to $1 / \mathrm{rev}$ \\
\hline$k_{\theta}$ & Reduced pitch frequency \\
\hline$k_{h}$ & Reduced plunge frequency \\
\hline$L$ & Likelihood function used in kriging interpolation \\
\hline$m, n$ & Number of previous time steps required to account for time history effects (see Eq. 4) \\
\hline M & Freestream Mach number \\
\hline $\bar{M}$ & Time-varying Mach number amplitude \\
\hline$M_{0}$ & Mean value of time-varying Mach number \\
\hline
\end{tabular}




\begin{tabular}{|c|c|}
\hline$N_{s p}$ & Number of sample points \\
\hline$N_{T}$ & Number of training cases \\
\hline$N_{t}$ & Total number of time steps for a test case \\
\hline$N_{x}$ & Number of input variables \\
\hline$N_{\text {basis }}$ & Number of basis functions associated with assumed polynomials in kriging \\
\hline$p_{k}$ & Fitting parameters in kriging \\
\hline q & Dynamical system state vector \\
\hline$r$ & Non-dimensional rotor blade radial location \\
\hline $\mathbf{r}_{\mathrm{krg}}(\mathbf{x})$ & Spatial correlation vector in kriging \\
\hline$R$ & Rotor radius \\
\hline $\mathbf{R}_{\mathrm{krg}}$ & Spatial correlation matrix used in kriging \\
\hline$R_{\mathrm{krg}}(\cdot)$ & Spatial correlation function in kriging \\
\hline$t_{0}, t_{f}$ & Initial and final time associated with CFD training cases \\
\hline $\mathbf{u}$ & Vector of external inputs \\
\hline$V_{0}$ & Mean value of freestream velocity \\
\hline$y$ & Output of interest \\
\hline$\hat{y}$ & Surrogate approximation of output of interest \\
\hline $\begin{array}{l}y_{\max }^{(j)}, y_{\min }^{(j)} \\
Z(\mathbf{x})\end{array}$ & $\begin{array}{l}\text { Maximum and minimum values of the exact response for the } j^{\text {th }} \text { test case } \\
\text { Stochastic process in kriging }\end{array}$ \\
\hline$\alpha_{\text {eff }}$ & Effective angle of attack (see Eq. (38)) \\
\hline$\Phi$ & Nonlinear mapping function \\
\hline$\hat{\Phi}$ & Surrogate mapping function \\
\hline$\hat{\Phi}_{q s}$ & Quasi-steady mapping function used for SBRF initialization \\
\hline$\lambda$ & Ratio between $M_{0}$ and $\bar{M}$ \\
\hline$\mu$ & Advance ratio \\
\hline$\omega_{1}$ & Oscillatory frequency corresponding to $1 / \mathrm{rev}$ \\
\hline$\omega_{\theta}$ & Higher harmonic pitch frequency \\
\hline$\omega_{h}$ & Oscillatory plunge frequency \\
\hline$\Omega$ & Rotational speed of the rotor \\
\hline$\theta$ & Airfoil pitch angle \\
\hline$\theta_{G}$ & Geometric pitch angle \\
\hline$\theta_{1}$ & Pitch amplitude corresponding to $1 /$ rev harmonic \\
\hline $\bar{\theta}$ & Higher harmonic pitch amplitude \\
\hline
\end{tabular}

\section{Introduction}

Dynamic stall is a strongly nonlinear unsteady aerodynamic phenomenon characterized by time-dependant separation and reattachment of the flow. ${ }^{1,2}$ The effects of this flow nonlinearity are critical in rotary-wing aeroelasticity applications in which dynamic stall can induce stall flutter ${ }^{3}$ and excessive vibratory loads that limit a helicopter's top speed and payload capacity. ${ }^{4}$ Currently, helicopter operational and performance boundaries must be constrained in order to avoid such adverse phenomena. Overcoming these limitations in the next generation of helicopter rotor designs will require active flow control and design optimization approaches that alleviate stall effects. Therefore, development of accurate dynamic stall models that are sufficiently efficient so as to be useful for computational aeroelasticity and active/passive design studies are required.

Typically, semi-empirical dynamic stall models are used in comprehensive rotor blade analysis codes due to their computational efficiency. The capabilities and limitations of several semi-empirical models employed in rotor blade analyses are described in Ref. 2. The most popular dynamic stall models are the 2-D ONERA model $^{5,6}$ and the 2-D Leishman-Beddoes model. ${ }^{2,7}$ As with all semi-empirical approaches, these models exhibit substantial deficiencies when analyzing different airfoils and/or arbitrary flow conditions for which experimental data is not available. The inability to accurately model 3-D effects such as swept flow is another important limitation associated with 2-D semi-empirical models. ${ }^{6}$ Furthermore, the ONERA and Leishman-Beddoes models are based on somewhat ad-hoc separation and reattachment criteria, and minor changes in these criteria can produce significant differences in aeroelastic response calculations.

Due to the limited applicability of semi-empirical approaches for modeling new rotor designs under 
arbitrary flow conditions, several ongoing research efforts are currently directed toward development of Computational Fluid Dynamics (CFD) methods for improved dynamic stall modeling. ${ }^{8}$ Although further development of CFD based dynamic stall models is required, ${ }^{9}$ CFD has been shown to result in significant improvements compared to semi-empirical models. ${ }^{8,10}$ A significant advantage facilitated by CFD methods is the ability to incorporate 3-D effects. Furthermore, CFD holds the potential for modeling active stall control methodologies - such as variable-droop leading edge airfoils, ${ }^{11,12}$ trailing edge flaps, ${ }^{13}$ and synthetic jets $^{14}$ - for which insufficient experimental data is available for empirical model development.

In order to fully assess the potential of active flow control and new rotor designs for alleviating dynamic stall effects, the improved fidelity afforded by CFD methods must be incorporated into comprehensive rotor blade analysis codes that are coupled with closed-loop control and design optimization frameworks. Although CFD based approaches have emerged as the most promising means of high-fidelity dynamic stall modeling, the excessive computational expense of such methods prevents their use in a variety of computational aeroelasticity and design optimization studies. Therefore, reduced-order models that retain the fidelity associated with CFD while exhibiting the computational efficiency of semi-empirical models must be developed. Reduced-order aerodynamic modeling refers to computationally efficient approximations of CFD results generated by extracting information from a limited number of full-order (CFD) solutions. Examples of such approaches include proper orthogonal decomposition (POD),${ }^{15,16}$ Volterra series, ${ }^{15,17,18}$ and surrogate based approaches. ${ }^{19-23}$ A major advantage of reduced-order modeling compared to empirical approaches is that the reduced-order models can be easily upgraded as the CFD solvers from which they are generated continue to improve.

Until recently, a nonlinear reduced-order modeling approach with the capabilities required for rotary-wing aeroelasticity computations had not been developed. In Ref. 24, the Surrogate Based Recurrence Framework (SBRF) approach to reduced-order aerodynamic modeling was introduced. It was shown in Ref. 24 that the SBRF method results in a time domain, fully unsteady model that accounts for flow nonlinearities, simultaneous pitch/plunge airfoil motions, and time-varying freestream Mach numbers. Furthermore, the SBRF approach resulted in an accurate reduced-order model when constructed with less than half of the CFD solutions utilized by a POD based model described in the literature. ${ }^{25}$ Therefore, the SBRF approach is suitable for rotor blade analysis and has been shown to compare favorably with existing reduced-order modeling methods. However, the study described in Ref. 24 was limited to transonic flow nonlinearities and simplified airfoil motions that are not representative of realistic helicopter rotor blade kinematics. Therefore, the objective of this study is to demonstrate the effectiveness of the SBRF approach for reduced-order dynamic stall modeling associated with airfoil motions representative of helicopter rotor blade dynamics. It is important to note that while the complex rotary-wing aerodynamic environment motivated the development of the model described in this paper, the approach is general and can be used in a variety of engineering applications involving nonlinear unsteady aerodynamics.

\section{Surrogate Based Reduced-Order Model}

Surrogates refer to computationally efficient approximations of expensive functions that are constructed by interpolating fitting data in the form of input/output combinations generated from a limited number of full-order computations. ${ }^{26-28}$ Once constructed, a surrogate is used in place of the expensive full-order analysis in order to predict at inputs that were not included in the initial set of fitting points. Typical surrogate prediction times are on the order of a fraction of a second, and therefore surrogates are ideal for reducedorder modeling. Although generating the fitting data may require significant computational resources, this initial cost is generally much less than computing repeated solutions from the expensive full-order analysis. Therefore, surrogate based approaches are appropriate for applications that entail numerous full-order analysis evaluations which would otherwise be unaffordable. A variety of computational aeroelasticity and design optimization applications involving CFD fall under this category.

In this study, CFD solutions for unsteady lift, moment, and drag due to an arbitrary airfoil motion represent the full-order analysis which is to be replaced by surrogates. The methodology for generating and utilizing the surrogates for time domain predictions are described next. 

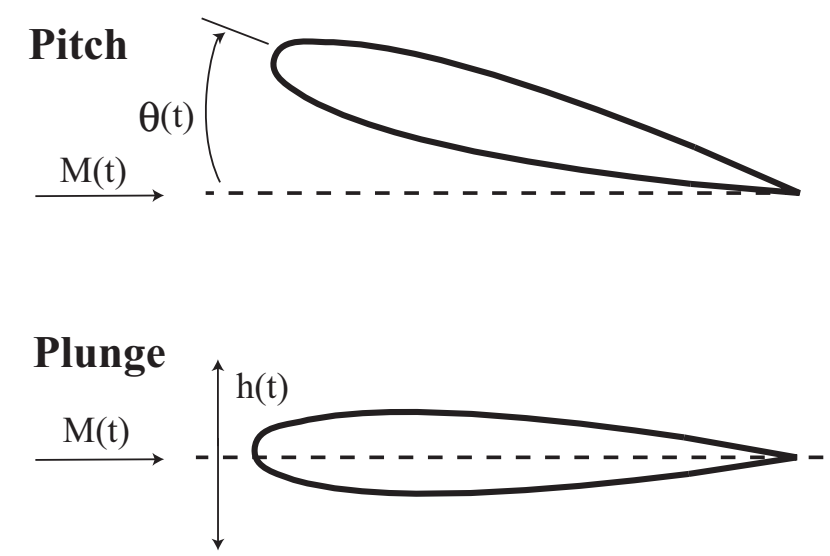

Figure 1. Airfoil pitch and plunge degrees of freedom with time-varying freestream Mach number

\section{II.A. Dynamical System Representation of the Input/Output Relationship}

The input/output relationship that is to be approximated by a surrogate model can be identified by formulating unsteady aerodynamic responses as dynamical systems; i.e. systems in which the output at any time $t$ is not only a function of the instantaneous input at $t$, but is also a function of the input time history. A general representation of a nonlinear time-invariant discrete time dynamical system is given by ${ }^{29-32}$

$$
\begin{aligned}
& \mathbf{q}(t)=G_{q}(\mathbf{q}(t-\Delta t), \mathbf{u}(t-\Delta t)) \\
& y(t)=G_{y}(\mathbf{q}(t))
\end{aligned}
$$

in which $\mathbf{q}$ is a vector containing the system states, $\mathbf{u}$ is the vector of external inputs to the system, $G_{q}$ is the one-step-ahead state transition function, $y(t)$ is the output of interest at some instant in time $t$, and $G_{y}$ is a function that maps the system states to the output.

In the context of unsteady aerodynamic modeling, the nonlinear system of equations described by Eq. (1) represents the discretized Navier-Stokes equations associated with CFD solvers. The state vector q consists of the flow states associated with the Navier-Stokes equations (i.e. density, velocity components, and energy) at each grid point in the spatially discretized computational domain. Therefore, the size of the state vector is proportional to the number of grid points and represents the order of the full-order model. Furthermore,

$$
y(t) \equiv C_{l}(t), C_{m}(t), \text { or } C_{d}(t)
$$

where $C_{l}(t), C_{m}(t)$, and $C_{d}(t)$ are the unsteady airfoil lift, moment, and drag coefficients respectively. The relevant external inputs are

$$
\mathbf{u}(t) \equiv\left[\begin{array}{lllllll}
\theta(t) & \dot{\theta}(t) & \ddot{\theta}(t) & \dot{h}(t) & \ddot{h}(t) & M(t) & \dot{M}(t)
\end{array}\right] .
$$

As shown in Fig. 1, $\theta(t), h(t)$, and $M(t)$ are the instantaneous pitch angle, plunge displacement, and freestream Mach number respectively. Time derivatives are denoted by $\dot{()} \equiv \frac{\partial()}{\partial t}$ and $\ddot{()} \equiv \frac{\partial^{2}()}{\partial t^{2}}$. The inputs in Eq. (3) were selected because it is known from linear aerodynamic theories that unsteady lift and moment are functions of these quantities, ${ }^{33}$ including $\dot{M}$ when modeling time-varying Mach numbers ${ }^{34}$ (which is a requirement for rotary-wing applications).

Equation (1)represents the computationally expensive full-order system which we seek to replace with a surrogate based reduced-order model. In order to generate a computationally efficient approximation of $y(t)$ using surrogate modeling, the dynamical system of interest must be replaced by an equivalent input/output functional relationship such that $y(t)$ corresponding to any input time history can be obtained without solving the nonlinear system of equations described by Eq. (1). It has been shown in Refs. 29-31 that when $\mathbf{q}$ is comprised of a finite number of states, the input/output relationship given by Eq. (4) is equivalent to the nonlinear system described by Eq. (1).

$$
y(t)=\Phi(\mathbf{u}(t), \mathbf{u}(t-\Delta t), \ldots, \mathbf{u}(t-m \Delta t), y(t-\Delta t), \ldots, y(t-n \Delta t))
$$


In Eq. (4), $\Phi$ is a function - possibly nonlinear - that maps the inputs to the output, and $m$ and $n$ are integers representing the number of previous external inputs and outputs required to account for time history effects. Selection of $m$ and $n$ is discussed in Section IV. Equation (4) is commonly referred to as a NARMAX (Nonlinear Auto Regressive Moving Average with eXogeneous inputs) model. ${ }^{29,31,32,35,36}$

It is important to note that the derivations described in Refs. 29 and 30 guarantee the validity of Eq. (4) only in the vicinity of an equilibrium state. An equilibrium state $\mathbf{q}_{0}$ is a state in which the system is at rest for an input sequence that has a constant value $\mathbf{u}_{0}$; i.e. $y(t)$ is constant for all $t$ when $\mathbf{q}=\mathbf{q}_{0}$ and $\mathbf{u}(t)=\mathbf{u}_{0}$. Therefore, according to Refs. 29 and 30, Eq. (4) is only guaranteed to be valid in the vicinity of $\mathbf{u}_{0}$. However, it was shown in Ref. 31 that Eq. (4) can also be used to describe a globally valid input/output relationship, and thus Eq. (4) is not restricted to modeling systems for $\mathbf{u}(t)$ close to $\mathbf{u}_{0}$. The globally valid model described in Ref. 31 is characterized by increased values of $m$ and $n$ required to capture time history effects compared to the locally valid model described in Refs. 29 and 30.

\section{II.B. Approximation of the Nonlinear Mapping Function}

Although Eq. (4) provides a qualitative representation of the input/output relationship associated with a nonlinear dynamical system, it is of little practical use for modeling unsteady aerodynamic responses because a closed-form expression for $\Phi$ is not available. However, $\Phi$ can be numerically evaluated, or sampled, by employing CFD as a black-box function that calculates lift, moment, and drag for a given airfoil motion time history. A surrogate mapping function $\hat{\Phi}$ can then be constructed by interpolating the sample data. Once constructed, the computationally efficient surrogate can be used to replace the full-order function $\Phi$. When modeling dynamical systems, the process of approximating the unknown function $\Phi$ is also referred to as system identification. ${ }^{32}$ The steps for generating the surrogate mapping function are summarized below, and additional details for each step are provided in the following subsections.

1. Use design of experiment (DOE) methods ${ }^{27,37}$ to select a limited number of training cases at which to conduct CFD simulations. Each training case consists of an airfoil undergoing simultaneous pitch/plunge oscillations, with either a fixed freestream Mach number, or time-varying Mach numbers if the surrogate is to be used for rotary-wing applications.

2. Generate aerodynamic response data for each training case using CFD, and then extract time domain sample data of the form dictated by Eq. (4) from the results. Each training case can be computed simultaneously using multiple processors. Therefore the time required to generate the initial fitting data can be decreased significantly through the use of parallel computation.

3. Construct a surrogate mapping function $\hat{\Phi}$ from the sampled data by employing kriging interpolation, ${ }^{38,39}$ which is a method well suited for approximating nonlinear functions.

\section{II.B.1. Selection of the Training Cases (DOE)}

In order to generate the input/output combinations required for interpolation, the unsteady aerodynamic responses corresponding to a limited number of training cases are obtained from CFD simulations. The training cases should be representative of the application for which the surrogate will be used. For instance, the training motions considered in this study correspond to simultaneous pitch/plunge oscillations with time-varying freestream Mach numbers since these cases are representative of those encountered in rotarywing aeroelasticity. The airfoil oscillations and time-varying Mach numbers used for training are defined by Eqs. (5) - (7).

$$
\begin{gathered}
\theta(t)=\theta_{G}-\theta_{1} \sin \left(\omega_{1} t\right)+\bar{\theta} \sin \left(\omega_{\theta} t\right) \\
h(t)=\bar{h} \cos \left(\omega_{h} t\right) \\
M(t)=M_{0}+\bar{M} \sin \left(\omega_{1} t\right)
\end{gathered}
$$

In Eq. (5), $\theta_{G}$ is the geometric pitch angle consisting of collective pitch and built-in twist components. The cyclic pitch inputs required for trim are represented by the oscillatory amplitude $\theta_{1}$ and the oscillatory frequency $\omega_{1}$ which corresponds to one period per revolution of the blade (i.e. $1 / \mathrm{rev}$ ). Note that including a $1 /$ rev cosine component representative of cyclic pitch ${ }^{4}$ is not expected to be important for the purposes of 


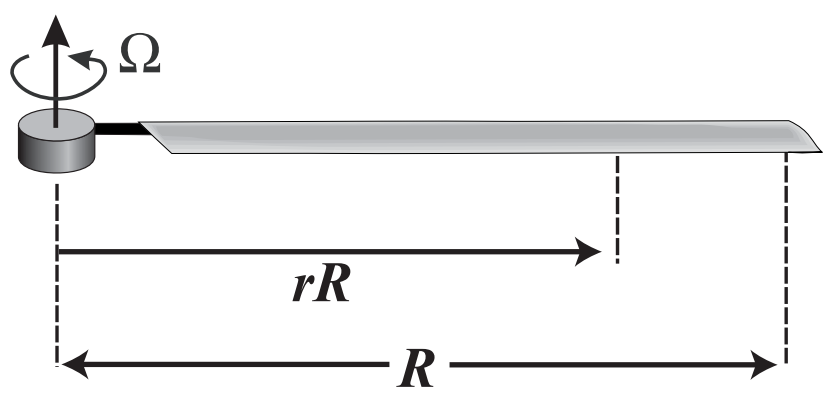

Figure 2. Typical rotor blade configuration.

constructing a surrogate. This is because the sum of a cosine and sine term of the same frequency can be written as an equivalent sine term, i.e:

$$
A \cos (\omega t)+B \sin (\omega t)=\sqrt{\left(A^{2}+B^{2}\right)} \sin (\omega t+\phi), \tan \phi=A / B .
$$

The amplitude $\theta_{1}$ in Eq. (5) accounts for the resultant amplitude $\sqrt{\left(A^{2}+B^{2}\right)}$, and as shown in Ref. 24, a surrogate trained on harmonic airfoil motions with $\phi=0$ can generate accurate predictions for harmonic external inputs with arbitrary values of $\phi$. This is because a sufficiently space-filling set of $\theta, \dot{\theta}$, and $\ddot{\theta}$ can be generated with $\phi=0$ such that the resulting surrogate can accurately predict at arbitrary pitch inputs corresponding to any $\phi .^{24}$ Therefore, including a $1 /$ rev cosine term, or $\phi$, in Eq. (5) is not expected to significantly contribute to the reduced-order model's accuracy. The final term in Eq. (5) accounts for higher harmonic pitch oscillations (i.e. $\omega_{\theta}>1 / \mathrm{rev}$ ) representative of elastic twist deformation.

Equation (7) is representative of the time-varying freestream Mach numbers associated with a helicopter rotor blade during forward flight. ${ }^{2}$ For a typical rotor blade depicted in Fig. 2, with rotational velocity $\Omega$ and radius $R$, the Mach number at the blade tip in hover (i.e. zero forward flight velocity) is given by

$$
M_{\Omega R}=\frac{\Omega R}{a} .
$$

The mean value and oscillatory amplitude in Eq. (7) are

$$
M_{0}=r M_{\Omega R}
$$

and

$$
\bar{M}=\mu M_{\Omega R}
$$

where $\mu$ is referred to as the advance ratio and is defined as the component of the forward flight velocity parallel to the hub plane of the rotor normalized by $\Omega R$.

The oscillatory frequencies in Eqs. (5) - (7) are given by

$$
\omega_{\theta}=\frac{k_{\theta} V_{0}}{b}, \omega_{h}=\frac{k_{h} V_{0}}{b}, \omega_{1}=\frac{k_{1} V_{0}}{b}
$$

where

$$
V_{0}=a M_{0}
$$

Furthermore,

$$
\omega_{1}=\Omega
$$

which gives

$$
k_{1}=\frac{\Omega b}{a M_{0}}=\frac{\Omega b}{a r M_{\Omega R}}=\frac{b}{R r} .
$$

Note that the unsteady aerodynamic loading is typically only modeled for $r>0.15$ in helicopter applications since the loads near the hub are relatively insignificant and their effects on the aeroelastic response of the blade can be neglected. Therefore, for given values of the non-dimensional airfoil semi-chord $b / R$ and $M_{\Omega R}$, 

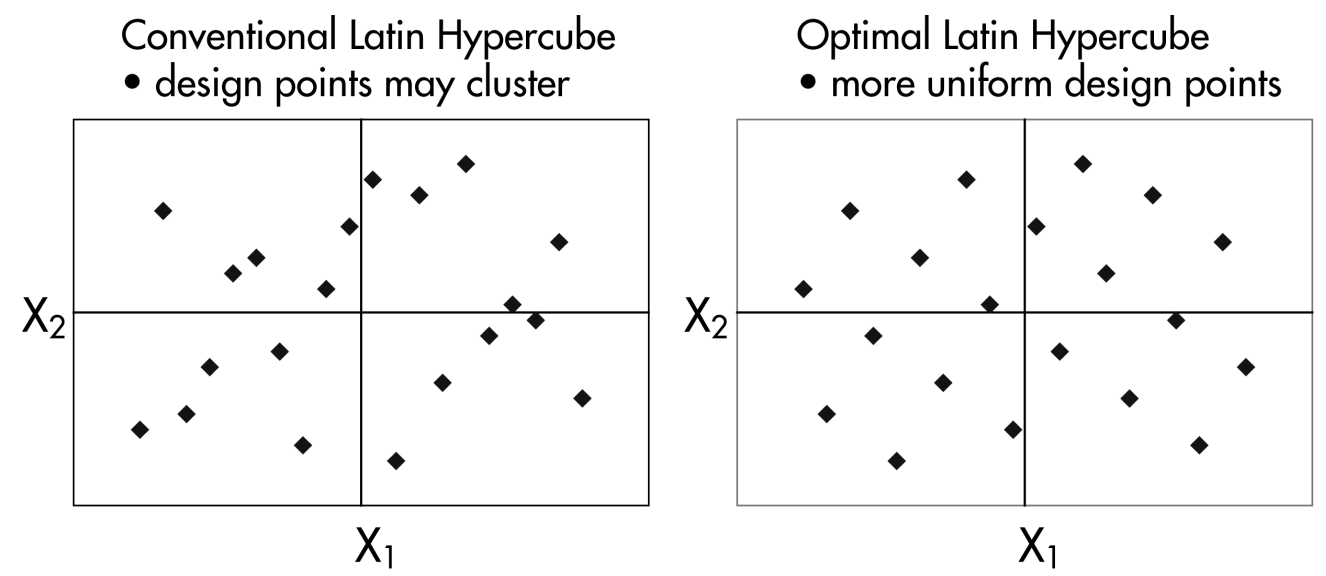

Figure 3. Conventional LH vs. optimal LH in 2-dimensional parameter space

a single training case given by Eqs. (5) - (7) is defined by 8 independent parameters: $\left(\theta_{G}, \theta_{1}, \bar{\theta}, k_{\theta}, \bar{h}, k_{h}, r, \mu\right)$.

Design of experiment (DOE) methods ${ }^{26,27}$ are used to select $N_{T}$ initial training cases at which to conduct CFD simulations. Each training case in the DOE consists of different combinations of the 8 independent parameters. When the initial data set is produced by a deterministic computer code, as opposed to a physical experiment or stochastic analysis, a given input will always yield the same output because there is no measurement error or other random sources of noise. Under these conditions, the DOE need only be space-filling ${ }^{37,40}$ so that all regions of the input parameter space are sampled.

A commonly used space-filling design is Latin hypercube sampling (LHS). ${ }^{41}$ In LHS, each input parameter is partitioned into $N_{T}$ equally spaced sections. Each input parameter is sampled once in each section, resulting in a column vector containing $N_{T}$ different values of the input parameter. The column vectors for each input parameter are arranged side by side into a matrix and the components of the vectors are then randomly reordered. The resulting matrix is known as a Latin hypercube. Since there are 8 independent parameters in this study, each hypercube is a $N_{T} \times 8$ matrix, in which each row corresponds to a different training case defined by the 8 input parameters. A major disadvantage of LHS is that training cases can cluster together due to the random combination of input parameters associated with each row of the Latin hypercube. To prevent this, optimal Latin hypercube (OLH) sampling is used in this study to ensure a more uniform (or space-filling) design of experiment. Optimal Latin hypercube sampling creates a more uniform design than conventional LHS by maximizing a spreading criteria, rather than randomly combining input parameters. Figure 3 illustrates the difference between a conventional Latin hypercube and an optimal Latin hypercube for a 2-dimensional parameter space. In this study, the OLH algorithm from the iSIGHT software package was used. ${ }^{42,43}$

\section{II.B.2. Extract Time Domain Sample Data}

For each training case, CFD is used to obtain time domain aerodynamic response data from $t_{0}$ to $t_{f}$, in increments of $\Delta t$. The sampled outputs and the corresponding inputs required to construct the surrogate mapping function $\hat{\Phi}$ are obtained from the CFD results. In this study, sample data from each training case is obtained for one rotor blade revolution, i.e.

$$
t_{f}-t_{0}=\frac{2 \pi}{\omega_{1}}
$$

From Eq. (4), the vector of inputs corresponding to a sampled output $y(t)$ is given by

$$
\mathbf{x}(t)=\left[\begin{array}{lllll}
\mathbf{u}_{j}(t) & \mathbf{u}_{j}(t-\Delta t) \ldots \mathbf{u}_{j}(t-m \Delta t) & y_{j}(t-\Delta t) \ldots y_{j}(t-n \Delta t
\end{array}\right] \text { for } j=1,2, \ldots, N_{T}
$$

where $y_{j}$ and $\mathbf{u}_{j}$ correspond to the $j^{\text {th }}$ training case and are given by Eqs. (2) and (3), and $\mathbf{x}$ is a vector of size $N_{x}=7(m+1)+n$, where the factor of 7 is due to the size of the external input vector given by Eq. (3).

For every training case in the OLH, fitting data of the form $(\mathbf{x}, y)$ is available at discrete time instants $t$, for $\left(t_{0}+\max [m, n] \Delta t\right) \leq t \leq t_{f}$. At time $t$ of the $j^{\text {th }}$ test case, $\mathbf{u}_{j}(t), \mathbf{u}_{j}(t-\Delta t), \ldots, \mathbf{u}_{j}(t-m \Delta t)$ are known 
from the prescribed motions defined by Eqs $5-7$, and the responses $y_{j}(t), y_{j}(t-\Delta t), \ldots, y_{j}(t-n \Delta t)$ are obtained from the CFD results. The sample data obtained from each of the $N_{T}$ training cases are combined into a single data set consisting of $N_{s p}$ total sample points, i.e. $\left(\mathbf{x}^{(i)}, y^{(i)}\right)$ for $i=1,2, \ldots, N_{s p}$. Note that generating the sample data can be expedited by using parallel computation since multiple training cases in the OLH can be simulated simultaneously.

\section{II.B.3. Construct Surrogate Mapping Function (Kriging Interpolation)}

The next step in constructing the reduced-order aerodynamic model is to approximate the nonlinear mapping function by interpolating the sampled data. The resulting surrogate mapping function, $\hat{\Phi}$, is used in place of the exact function in order to approximate full-order unsteady airload predictions corresponding to arbitrary inputs. In order to be useful for modeling nonlinear flow effects, the interpolation method must be well suited to approximating nonlinear functions and should not require a priori assumptions on the form of the function that is to be approximated. Kriging interpolation ${ }^{38,39}$ satisfies these conditions and is therefore used to generate the surrogate mapping functions in this study.

In kriging, the unknown function of interest, $\Phi(\mathbf{x})$, is assumed to be a random variable of the form

$$
\Phi(\mathbf{x})=f(\mathbf{x})+Z(\mathbf{x})
$$

where $f(\mathbf{x})$ is an assumed function (usually a low-order polynomial) and $Z(\mathbf{x})$ is a stochastic (random) process which is assumed to be Gaussian with zero mean and variance $\sigma_{v a r}^{2}$. The regression model $f(\mathbf{x})$ can be thought of as a globally valid trend function, while $Z(\mathbf{x})$ accounts for local deviations from $f(\mathbf{x})$ which ensure that the kriging model interpolates the sample points exactly. The local deviations dictated by the sample points enable the kriging predictor to approximate nonlinear behavior regardless of the exact function's form. Note that although $\Phi(\mathbf{x})$ is deterministic, rather than the stochastic process assumed in Eq. (18), kriging interpolation is still applicable. The assumption that $\Phi(\mathbf{x})$ is a random process is made because the deviation from the regression model can resemble a realization of a stochastic process. ${ }^{38}$

The covariance matrix of $Z(\mathbf{x})$, which is a measure of how strongly correlated two points are, is given by

$$
\operatorname{Cov}\left[Z\left(\mathbf{x}^{(i)}\right), Z\left(\mathbf{x}^{(j)}\right)\right]=\sigma_{v a r}^{2} \mathbf{R}_{\mathrm{krg}}
$$

where each element of the $N_{s p} \times N_{s p}$ correlation matrix $\mathbf{R}_{\mathrm{krg}}$ is given by

$$
\left(R_{\mathrm{krg}}\right)_{i j}=R_{\mathrm{krg}}\left(\mathbf{x}^{(i)}, \mathbf{x}^{(j)}\right)
$$

and $R_{\text {krg }}$ is a user defined spatial correlation function $(\mathrm{SCF})$. In this study,

$$
R_{\mathrm{krg}}\left(\mathbf{x}^{(i)}, \mathbf{x}^{(j)}\right)=\prod_{k=1}^{N_{x}} \max \left[0,1-p_{k}\left|x_{k}^{(i)}-x_{k}^{(j)}\right|\right],
$$

where $x_{k}^{(i)}$ is the $k^{\text {th }}$ component of the $i^{\text {th }}$ sample input given by Eq. (17). This SCF was selected because the resulting correlation matrices were not ill-conditioned, which is an issue that may arise with kriging. ${ }^{39}$ As two points move closer to each other, $\left|x_{k}^{(i)}-x_{k}^{(j)}\right| \rightarrow 0$, and Eq. (21) approaches unity which is the maximum value of the SCF. Therefore, the SCF recovers the intuitive property that the closer two points are to each other, the greater the correlation between the points.

The fitting parameters $p_{k}$ are unknown correlation parameters which need to be determined. In order to determine these parameters, the form of $f(\mathbf{x})$ needs to be chosen. In this study, $f(\mathbf{x})$ is assumed to be a $2^{\text {nd }}$ order polynomial given by

$$
f(\mathbf{x})=f_{x}^{\mathrm{T}} \boldsymbol{\beta}
$$

where $f_{x}^{\mathrm{T}}$ is a $1 \times N_{\text {basis }}$ vector, $N_{\text {basis }}$ is the number of basis functions associated with the $2^{\text {nd }}$ order polynomial, and $\beta$ is a $N_{\text {basis }} \times 1$ vector of coefficients. Similarly, $\mathbf{F}(\mathbf{x})$ can be defined as an $N_{s p} \times N_{\text {basis }}$ matrix where the $i^{t h}$ row corresponds to the evaluation of the $N_{\text {basis }}$ functions at the $i^{\text {th }}$ sample point.

In order to find $p_{k}$, the generalized least square estimates of $\boldsymbol{\beta}$ and $\sigma_{v a r}^{2}$, denoted by $\hat{\boldsymbol{\beta}}$ and $\hat{\sigma}_{v a r}^{2}$ respectively, are employed: ${ }^{38,39}$

$$
\hat{\boldsymbol{\beta}}=\left(\mathbf{F}^{\mathrm{T}}\left(\mathbf{R}_{\mathrm{krg}}\right)^{-1} \mathbf{F}\right)^{-1} \mathbf{F}^{\mathrm{T}}\left(\mathbf{R}_{\mathrm{krg}}\right)^{-1} \mathbf{y}
$$


and

$$
\hat{\sigma}_{v a r}^{2}=\frac{(\mathbf{y}-\mathbf{F} \hat{\boldsymbol{\beta}})^{\mathrm{T}}\left(\mathbf{R}_{\mathrm{krg}}\right)^{-1}(\mathbf{y}-\mathbf{F} \hat{\boldsymbol{\beta}})}{N_{s p}}
$$

where $\mathbf{y}$ is a $N_{s p} \times 1$ vector of observed function outputs at the sampled inputs

With $\hat{\sigma}_{\text {var }}^{2}$ and $\hat{\boldsymbol{\beta}}$ known, $p_{k}$ are found such that a likelihood function is maximized. ${ }^{39,44}$ The likelihood function, given in Eq. 25, represents the probability that the stochastic process $\Phi(\mathbf{x})$ produced the sampled data points in $\mathbf{y}$. Since the stochastic process associated with kriging has been assumed to be Gaussian, one seeks the set $p_{k}$ that maximizes the probability that the sample points have been drawn from a Gaussian process.

$$
L\left(\mathbf{y}, p_{k}\right)=-\frac{N_{s p} \ln \left(\hat{\sigma}_{v a r}^{2}\right)+\ln \left|\mathbf{R}_{\mathrm{krg}}\right|}{2}
$$

The maximum likelihood estimates (MLE's) of $p_{k}$ represent the fitting parameters that are most consistent with the sampled data. Any values of $p_{k}$ would result in a surrogate which interpolates the sample points exactly, but the "best" kriging surrogate is found by maximizing the likelihood function.

With all parameters known, the kriging approximation of a function $\Phi(\mathbf{x})$ is given by ${ }^{38}$

$$
\hat{\Phi}(\mathbf{x})=f_{x}^{\mathrm{T}} \hat{\boldsymbol{\beta}}+\mathbf{r}_{\mathrm{krg}}^{\mathrm{T}}(\mathbf{x})\left(\mathbf{R}_{\mathrm{krg}}\right)^{-1}(\mathbf{y}-\mathbf{F} \hat{\boldsymbol{\beta}})
$$

where

$$
\mathbf{r}_{\mathrm{krg}}(\mathbf{x})=\left[R_{\mathrm{krg}}\left(\mathbf{x}, \mathbf{x}^{(1)}\right), R_{\mathrm{krg}}\left(\mathbf{x}, \mathbf{x}^{(2)}\right), \ldots, R_{\mathrm{krg}}\left(\mathbf{x}, \mathbf{x}^{\left(N_{s p}\right)}\right)\right]^{\mathrm{T}} .
$$

The column vector $\mathbf{r}_{\mathrm{krg}}(\mathbf{x})$ of length $N_{s p}$ is the correlation vector between an arbitrary prediction point $\mathbf{x}$ and the sampled inputs, $\mathbf{x}^{(1)}, \ldots, \mathbf{x}^{\left(N_{s p}\right)}$. The kriging predictor given by Eq. (26) represents the optimal predictor in the sense that, among all admissible predictors, Eq. (26) results in the minimum mean square error with respect to the assumed stochastic process $\Phi(\mathbf{x}){ }^{38}$ Note that although the kriging predictor is derived as an approximation to a stochastic process, Eq. (26) is a deterministic function. Therefore, kriging has been used extensively in approximating deterministic computer models. ${ }^{39}$ The kriging surrogates were created with a freely available MATLAB toolbox ${ }^{45}$ and the DIRECT optimization algorithm ${ }^{46,47}$ was used to conduct a global search for the maximum likelihood parameter estimates.

\section{II.C. Time Domain Predictions Using a Surrogate Based Recurrence Framework}

The surrogate mapping function can be used for discrete time domain predictions when provided with arbitrary instances of the input vector $\mathbf{x}(t)$ given by Eq. (17). However, $n$ components of $\mathbf{x}$ correspond to previous values of the unsteady aerodynamic response, which will not be known for arbitrary inputs. Only the surrogate's approximation of the previous responses will be available. Therefore, an approximate input vector, $\hat{\mathbf{x}}$, consisting of the surrogate's predictions of the previous responses must be used in order to obtain predictions at an arbitrary input. The approximate input vector is

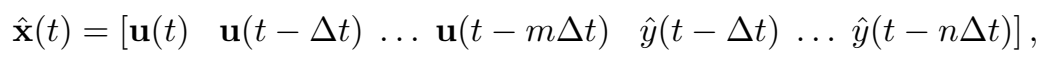

where the approximate previous responses $\hat{y}$ are obtained from the surrogate's predictions at previous time steps. The development of the reduced-order aerodynamic model is completed by utilizing the surrogate mapping function within the recurrence framework depicted in Fig. 4. The term "recurrence" refers to the property that the approximate solutions, $\hat{y}$, are fed back and used as inputs to $\hat{\Phi}$ for the prediction at the current time. Therefore, the approximate aerodynamic responses obtained from the surrogate based recurrence framework $(\mathrm{SBRF})$ can be written as

$$
\hat{y}(t)=\hat{\Phi}(\hat{\mathbf{x}}(t)) .
$$

The process depicted in Fig. 4 is marched forward in increments of $\Delta t$ until the approximate solution at some final time is obtained. Note that guesses for the $n$ previous responses are needed to initialize the SBRF. However, as shown in Ref. 24, the accuracy of the initial guesses is not critical since the initial transients in the SBRF's predictions die out as time is marched forward. In summary, the SBRF accounts for time history effects associated with unsteady flows by employing a recurrence solution methodology, while flow nonlinearities are modeled by utilizing a kriging approximation of the full-order mapping function. 


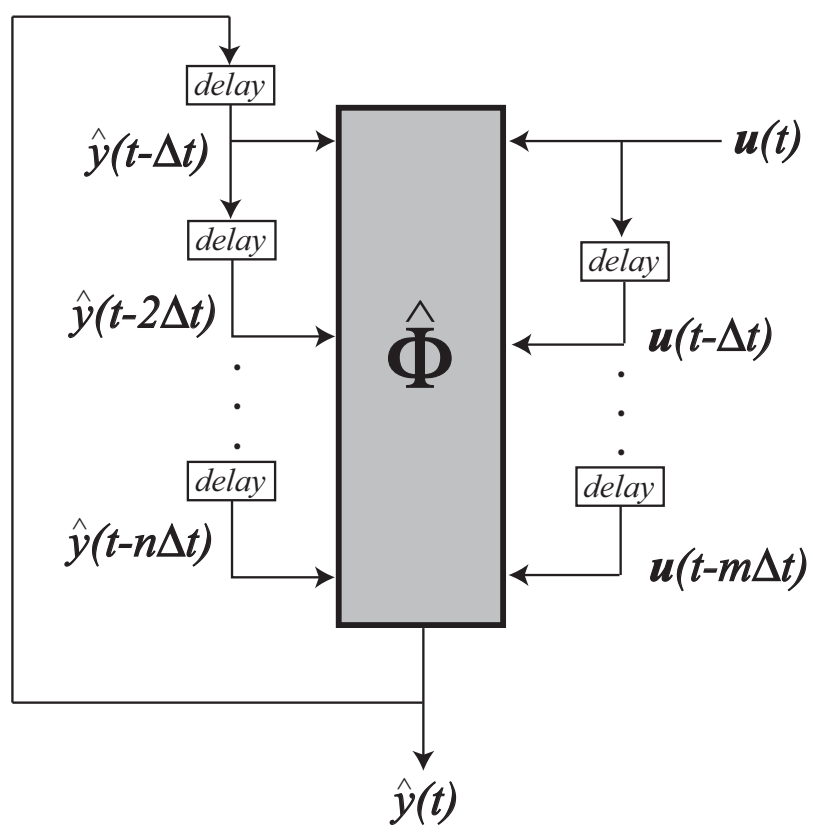

Figure 4. Surrogate based recurrence framework (SBRF).

\section{CFD Solvers}

In this study, the full-order aerodynamic solutions were generated by the compressible Navier-Stokes CFD code OVERFLOW version 2.0y, as modified during the DARPA Helicopter Quieting Program. ${ }^{48}$ Additional CFD results based on the commercially available code CFD $++{ }^{49,50}$ developed by METACOMP Technologies were used to validate the implementation of OVERFLOW for the required combined airfoil and freestream Mach number oscillations. Brief descriptions of the two CFD solvers are provided next.

\section{III.A. OVERFLOW}

The OVERFLOW code, originally developed by NASA, uses overset structured grids and has been validated for a wide variety of problems by academia, government, and industry for studies in dynamic stall, ${ }^{11}$ fixed wing, ${ }^{51}$ rotary wing, ${ }^{52}$ and spacecraft applications. ${ }^{53}$ The Spalart-Allmaras and Kinetic Eddy Simulation $(\mathrm{KES})^{54}$ turbulence models were used with 4 th order central differencing in this study. The near body grid was created using Chimera Grid Tools. ${ }^{55}$ The grid is of dimensions $295 \times 41$ in the chordwise and normal direction as illustrated in Fig. 5(a). The normal direction has initial spacing of $y^{+}$less than one for the maximum Reynolds number considered, and a total normal spacing of half a chord. The complex airfoil motion is created using the XML interface. ${ }^{56}$ The freestream velocity was set to correspond to the mean Mach number from rotor rotation $\left(M_{0}\right)$. The azimuthal variation in velocity $(\bar{M})$ was modeled by translating the airfoil in a sinusoidal fashion. For each main cycle of motion, 17500 time steps were used with 8 Newton sub-iterations to achieve 2 nd order accuracy in time. The location of the level one off body grid was adapted every 250 time steps to the airfoil motion. The total number of grid points varied during the airfoil motion computations, with a mean value of the variation corresponding to approximately 150000 points.

\section{III.B. CFD ++}

The CFD ++ code is capable of solving the compressible unsteady Reynolds-Averaged Navier-Stokes equations. It uses a unified grid methodology that can handle a variety of structured, unstructured, multi-block meshes and cell types, including patched and overset grid features. Spatial discretization of the Navier-Stokes equations is based on a second order multi-dimensional Total Variation Diminishing (TVD) scheme. ${ }^{57}$ For temporal discretization an implicit algorithm with dual time-stepping and multigrid acceleration is used. Dual time-stepping schemes are constructed by appending a pseudo time derivative term and using subiterations for improved accuracy. Multigrid acceleration employs a hierarchy of coarsening grids to speed the 


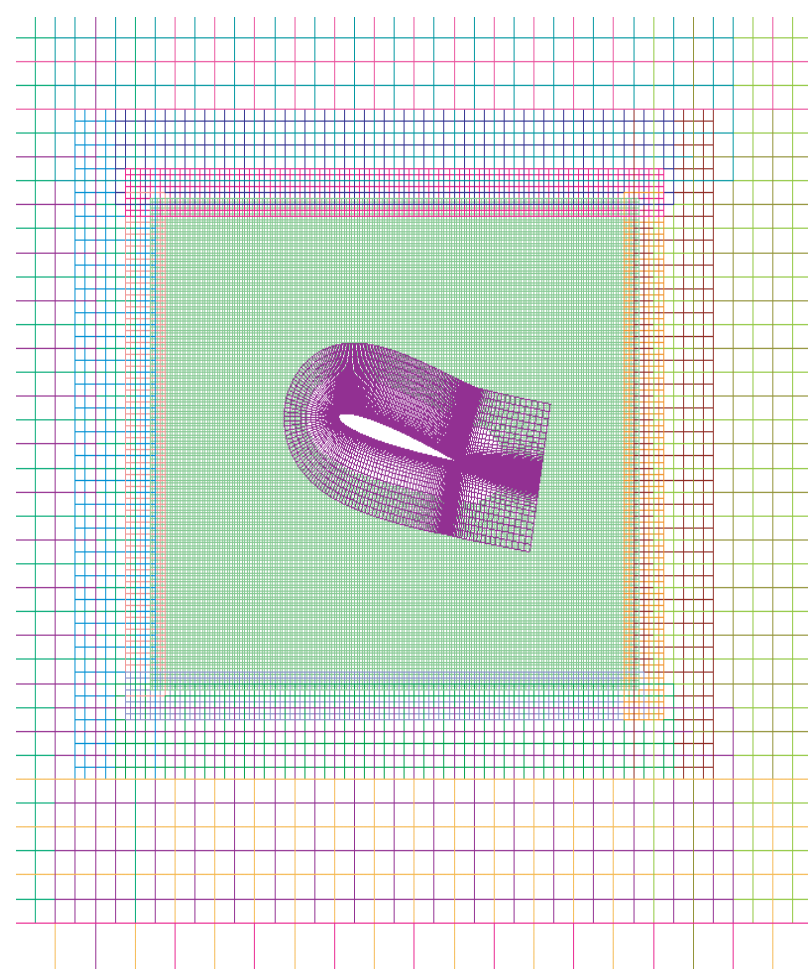

(a) OVERFLOW Grid

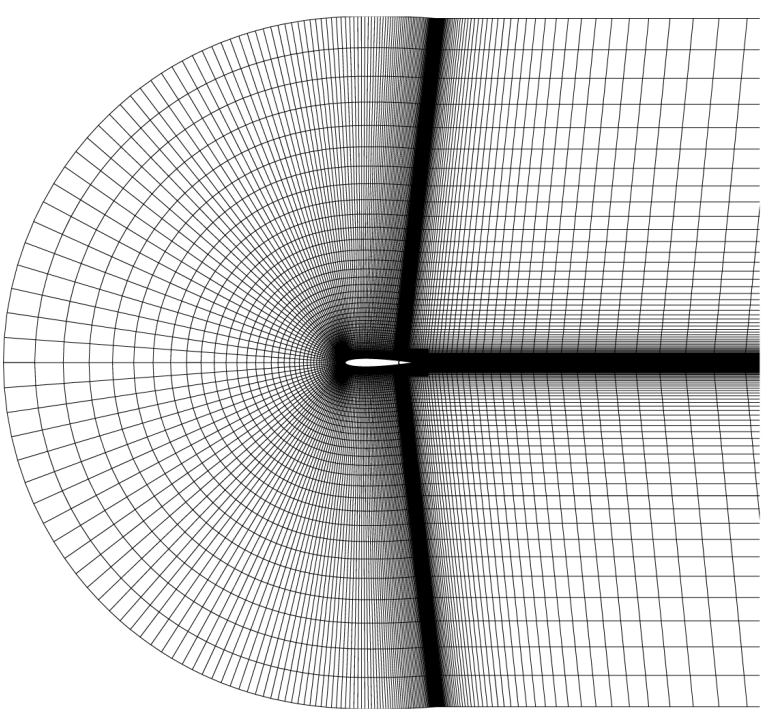

(b) $\mathrm{CFD}++$ Grid.

Figure 5. Computational grids used for OVERFLOW and CFD ++ calculations.

convergence. Several turbulence models are available in $\mathrm{CFD}++$, ranging from 1-equation to 3-equation transport models.

All CFD ++ computations are carried out by solving RANS equations and assuming fully turbulent flow. The computational domain is a C-grid, as shown in Fig. 5(b), with the far field boundary extending to 50 chord lengths in all directions. A distance of $30-50$ chord lengths is typically used in order to minimize the effects of numerical reflection from the far field boundary. The grids are clustered at the airfoil wall boundaries such that the dimensionless distance $y^{+}$of the first grid point off the wall is less than 1 and the equations are directly solved to the walls without assuming any wall functions. The Spalart-Allmaras turbulence model is used in all $\mathrm{CFD}++$ computations.

\section{Results}

The effectiveness of the SBRF approach is demonstrated by comparing against full-order CFD predictions for unsteady lift, moment, and drag coefficients associated with oscillating airfoils subject to fixed and timevarying freestream Mach numbers. In this study, $m=0$ and $n=2$ were used for all results since these values resulted in accurate approximations of time history effects in Ref. 24. In order to initialize the SBRF, responses corresponding to $n=2$ previous time-steps are required as inputs to the surrogate mapping function. These initial responses were obtained from $2^{\text {nd }}$ order polynomial response surfaces ${ }^{27}$ that were only a function of the external inputs (i.e. no previous response inputs). Therefore, the predictions of the previous response quantities required to initialize the SBRF are given by

$$
\hat{y}(t-i \Delta t)=\hat{\Phi}_{q s}(\mathbf{u}(t-i \Delta t)) \text { for } i=1, \ldots, n
$$

where $\hat{\Phi}_{q s}$ denotes that these response surfaces can be thought of as quasi-steady approximations since they are only functions of the instantaneous external inputs, and therefore have no mechanism to account for time history effects. After the initial $n$ time steps, the previous responses required as inputs are obtained from the SBRF feedback mechanism depicted in Fig. 4. As shown in Ref. 24, the accuracy of the initialization values is not critical since the resulting transients die out as time is marched forward and the SBRF predictions 
Table 1. Pitching Amplitudes and Reduced Frequencies for Training and Testing the SBRF ( $M=0.3$ )

\begin{tabular}{ccccc}
\hline \hline Cases & $\theta_{G}$ (degrees) & $\bar{\theta}$ (degrees) & $k_{\theta}$ & \# of cases \\
\hline Training & $15,10,5,0,-6$ & $10,5,2.5$ & $0.1,0.03$ & 30 \\
Test & $15,10,5,0,-6$ & $10,5,2.5$ & 0.05 & 15 \\
\hline \hline
\end{tabular}

Table 2. SBRF errors for 15 test cases corresponding to simplified pitch oscillations and $M=0.3$

\begin{tabular}{ccc}
\hline \hline Error in $\boldsymbol{C}_{\boldsymbol{\imath}}$ & Error in $\boldsymbol{C}_{\boldsymbol{m}}$ & Error in $\boldsymbol{C}_{\boldsymbol{d}}$ \\
\hline $0.6-3.3 \%($ avg. $=2.2 \%)$ & $1.4-3.8 \%($ avg. $=2.6 \%)$ & $0.9-3.1 \%($ avg. $=2.1 \%)$ \\
\hline \hline
\end{tabular}

quickly reach a periodic solution.

The following error metric was used to quantify the accuracy of the SBRF approach relative to CFD:

$$
E^{(j)}=100 \times \frac{\frac{1}{N_{t}} \sum_{i=1}^{N_{t}}\left|\hat{y}\left(t_{i}\right)-y\left(t_{i}\right)\right|}{y_{\max }^{(j)}-y_{\min }^{(j)}}
$$

In Eq. (31), $E^{(j)}$ is a relative error measure corresponding to the $j^{\text {th }}$ test case, the numerator corresponds to the average error over the total number of time steps, and the denominator represents the range of the exact response. Thus, Eq. (31) represents the average error in the SBRF relative to the peak-to-peak value of the exact response.

\section{IV.A. Simplified Pitch Oscillations Subjected to a Fixed Freestream Mach Number}

The effectiveness of the SBRF approach for modeling dynamic stall effects caused by excessive angles of attack is illustrated in this section by considering an SC-1095 airfoil oscillating in pitch at Mach 0.3. The simplified pitching motions are given by $\theta(t)=\theta_{G}+\bar{\theta} \sin \left(\omega_{\theta} t\right)$. The full-order solutions were obtained from OVERFLOW with the KES turbulence model. The SBRF was trained on CFD data corresponding to 30 pitching motions, and the reduced-order predictions were tested by comparing with CFD predictions for 15 airfoil motions that were not included in the set of training motions. Note that the full-order training cases correspond to existing results from a previous study, and were not generated using space-filling DOE methods. The pitching amplitudes and reduced frequencies for the training and test cases are provided in Table 1.

The errors for the 15 test cases are provided in Table 2. The maximum error for all cases was 3.8\%, which indicates that the SBRF accurately approximated the full-order aerodynamic responses for all test cases. Comparisons between the SBRF predictions and the OVERFLOW solutions for 4 of the 15 test cases are shown in Figs. 6 - 8. In Figs. 6 - 8, the arrows indicate if the angle of attack is increasing or decreasing. In all 4 cases depicted in Fig. 7 , the OVERFLOW predictions exhibit large drops in moment coefficient for certain portions of the airfoil motion associated with increasing angles of attack. This behavior is indicative of the strongly nonlinear behavior associated with dynamic stall. Overall, there is excellent agreement - no more than 3\% error - between the SBRF and OVERFLOW predictions of the nonlinear phenomena depicted in Figs. $6-8$. These results demonstrate that the SBRF can accurately approximate CFD predictions of the highly nonlinear, hysteretic behavior corresponding to unsteady sectional lift, moment, and drag under dynamic stall conditions. Note that the SBRF required a fraction of a second to generate the predictions for the test airfoil motions, while the CFD solutions required several hours of simulation time. The level of agreement between the SBRF and CFD for the 11 test cases that are not shown was similar to the results shown in Figs. 6 - 8. 


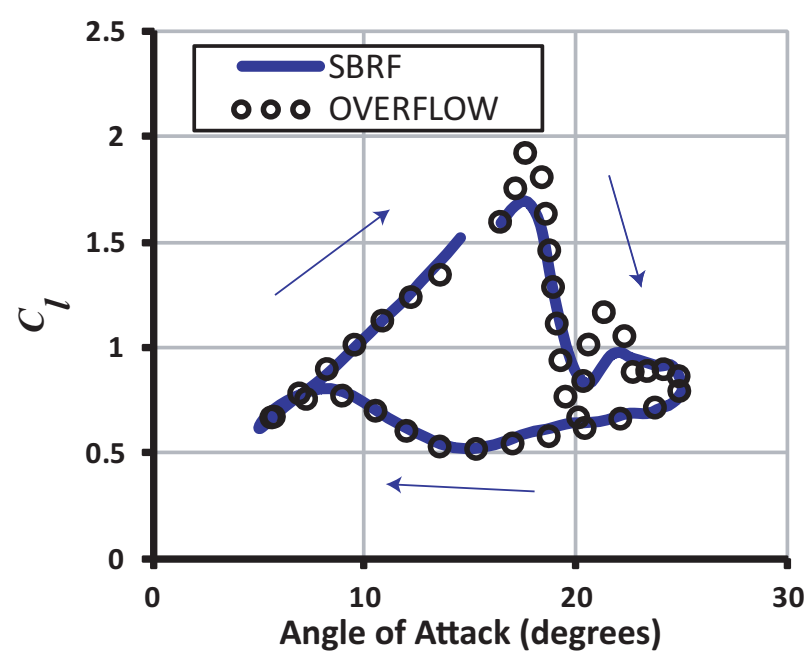

(a) $\theta_{G}=15^{\circ}, \bar{\theta}=10^{\circ}(3.0 \%$ error $)$

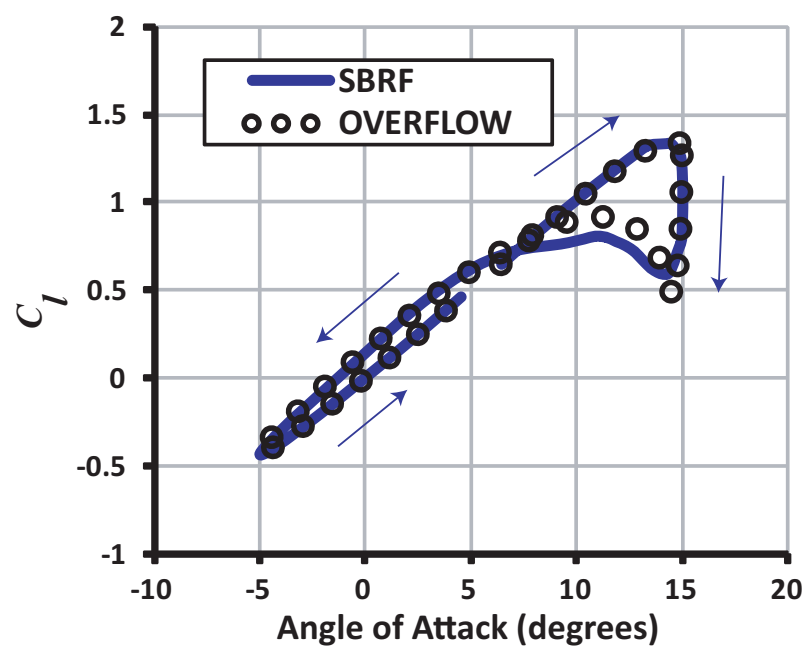

(c) $\theta_{G}=5^{\circ}, \bar{\theta}=10^{\circ}(2.1 \%$ error $)$

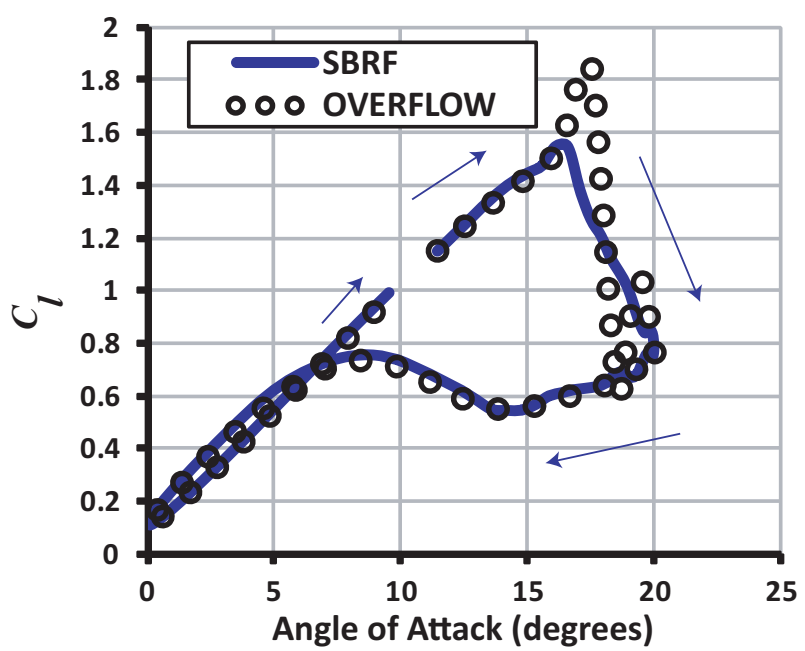

(b) $\theta_{G}=10^{\circ}, \bar{\theta}=10^{\circ}(2.3 \%$ error $)$

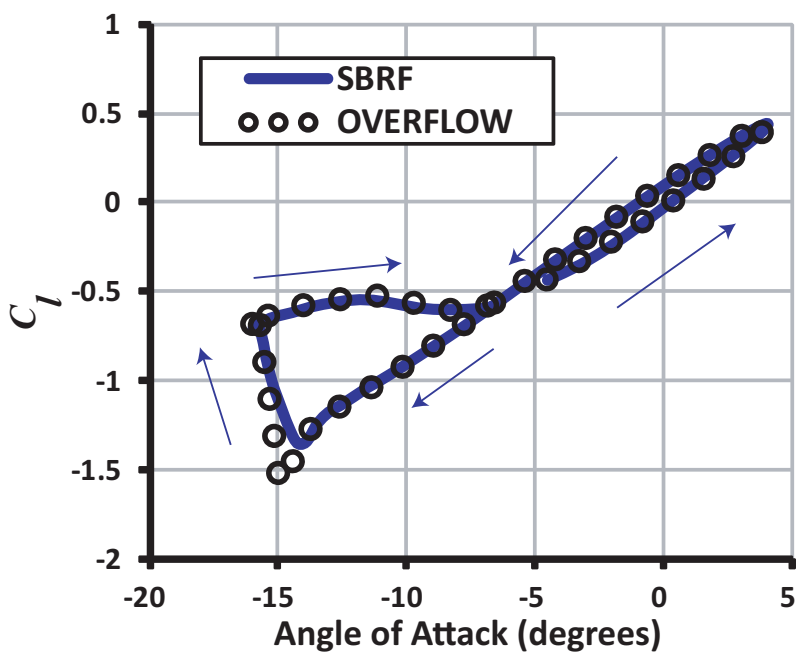

(d) $\theta_{G}=-6^{\circ}, \bar{\theta}=10^{\circ}(1.2 \%$ error $)$

Figure 6. Unsteady lift coefficients under dynamic stall conditions for a pitching airfoil $M=0.3, k_{\theta}=0.05$.

\section{IV.B. Combined Pitch/Plunge Oscillations Subjected to Time-Varying Mach Numbers}

In order to be effective for rotary-wing aeroelasticity applications, the SBRF approach must accurately approximate dynamic stall effects corresponding to combined pitch/plunge oscillations and time-varying freestream Mach numbers. Furthermore, while the results in Section IV.A illustrate the effectiveness of the SBRF for modeling flow separation due to excessive angles of attack - as in retreating blade stall cases dynamic stall on a helicopter rotor can also be caused by shock induced separation on the advancing side of the rotor. ${ }^{2}$ Therefore, cases corresponding to realistic airfoil motions as well as advancing and retreating side stall are considered in order to fully asses the applicability of the SBRF approach for helicopter rotor blade analysis. Validation results for the OVERFLOW implementation of combined pitch/plunge airfoil motion subject to time-varying Mach numbers, followed by reduced-order dynamic stall results are provided next.

All time-varying Mach number cases in this study correspond to a NACA 0012 airfoil with the following rotor parameters: $b / R=0.0275, M_{\Omega R}=0.64$, and $r=0.75$. These parameters are consistent with a model representative of the $75 \%$ spanwise station of an MBB BO-105 helicopter rotor. The $75 \%$ spanwise station was considered in this study since it is sufficiently outboard such that the advancing side Mach numbers are high enough for shock induced separation to occur. From Eqs. (10) and (15), $M_{0}=0.48$ and $k_{1}=0.037$ for all cases. The Spalart-Allmaras turbulence model was used in all OVERFLOW computations associated with combined pitch/plunge airfoil motion under time-varying Mach number conditions. 


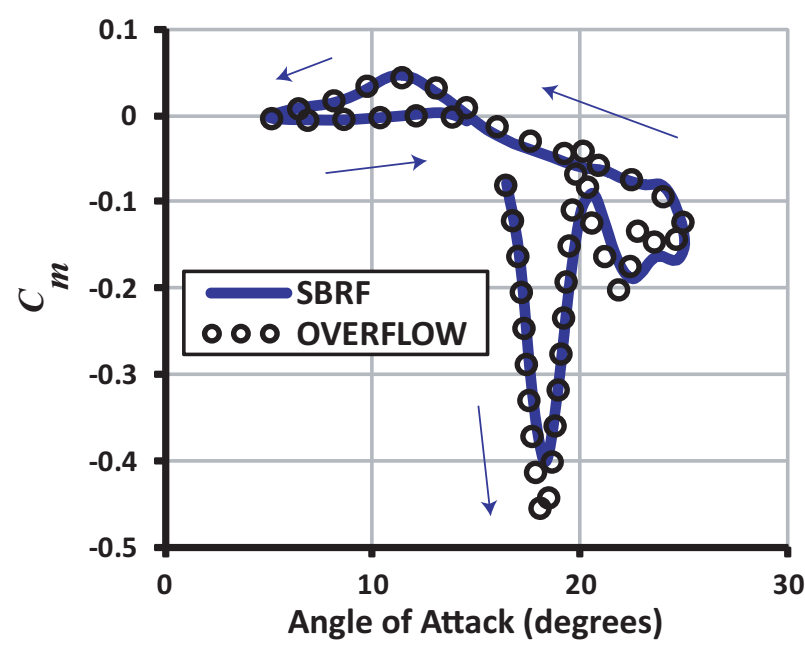

(a) $\theta_{G}=15^{\circ}, \bar{\theta}=10^{\circ}(2.1 \%$ error $)$

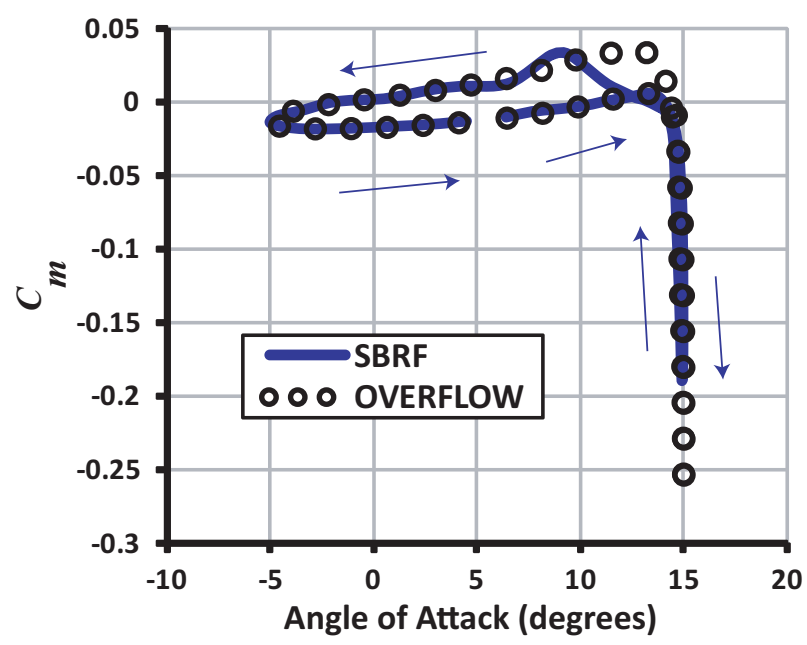

(c) $\theta_{G}=5^{\circ}, \bar{\theta}=10^{\circ}(2.4 \%$ error $)$

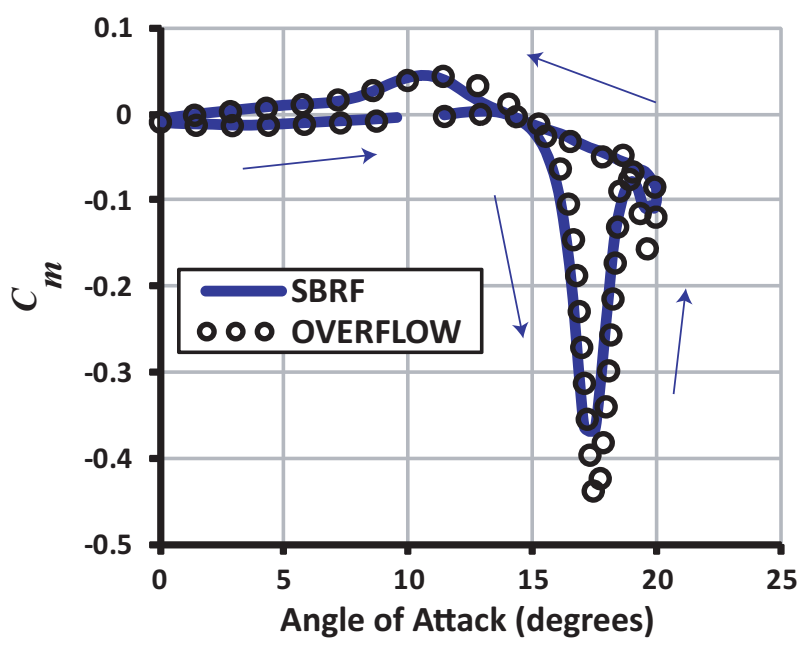

(b) $\theta_{G}=10^{\circ}, \bar{\theta}=10^{\circ}(1.7 \%$ error $)$

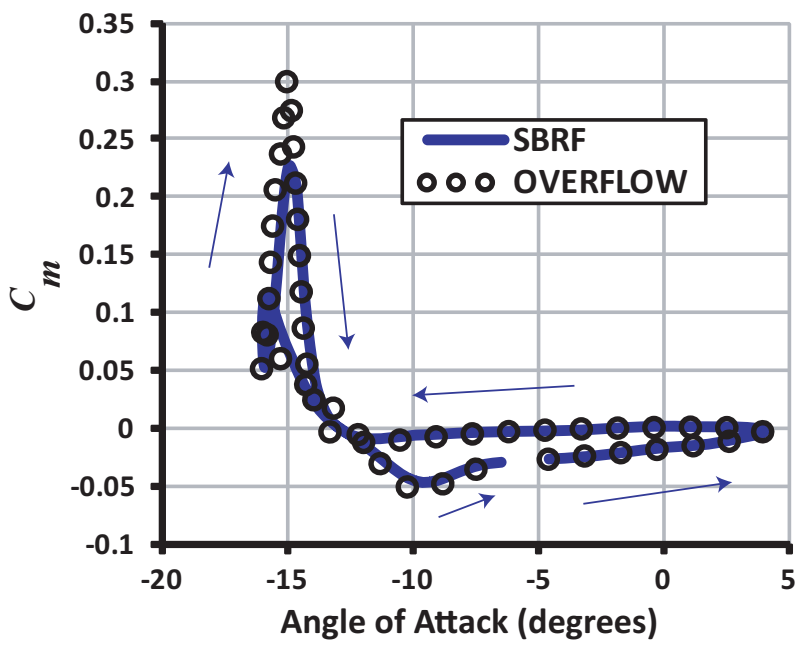

(d) $\theta_{G}=-6^{\circ}, \bar{\theta}=10^{\circ}(2.2 \%$ error $)$

Figure 7. Unsteady moment coefficients under dynamic stall conditions for a pitching airfoil $M=0.3, k_{\theta}=0.05$.

\section{IV.B.1. Validation of the OVERFLOW Implementation}

The implementation of OVERFLOW for computations associated with oscillating airfoils subject to timevarying Mach numbers was validated by comparing with $\mathrm{CFD}++$, which was utilized in Ref. 24 for similar cases. The CFD++ implementation of time-varying freestreams was verified by considering unsteady aerodynamic loads for a statically pitched airfoil subject to sinusoidal Mach number variations for incompressible flow, as in Ref. 58. In Ref. 58, theoretical results based on Isaacs' theory and indicial function Arbitrary Motion Theory (AMT) were compared to CFD simulations obtained with an Euler code. This comparison is shown in Fig. 9. The magnitude of freestream oscillation is characterized by $\lambda$, which represents the ratio between the amplitudes of time varying freestream velocity to the constant freestream velocity. The CFD++ Navier-Stokes solutions based on the Spalart-Allmaras turbulence model and a fully turbulent flow assumption are superimposed onto Fig. 9. Clearly, the implementation of time-varying Mach number calculations in CFD++ results in excellent agreement with the models considered in Ref. 58 for two cases of freestream oscillation amplitude, $\lambda=0.4$ and $\lambda=0.8$.

Since the CFD++ solutions agree with the models described in Ref. 58, the implementation of OVERFLOW for the required airfoil and freestream oscillations was verified by comparing OVERFLOW and $\mathrm{CFD}++$ for several cases consisting of various combinations of simultaneous pitch/plunge oscillations and time-varying Mach numbers. The comparisons for the unsteady lift, moment, and drag coefficients are shown in Fig. 10 for two cases. For both cases, $\theta_{G}=7.4^{\circ}, \theta_{1}=2.3^{\circ}, k_{1}=0.037, \bar{\theta}=7.4^{\circ}, k_{\theta}=0.20$, 


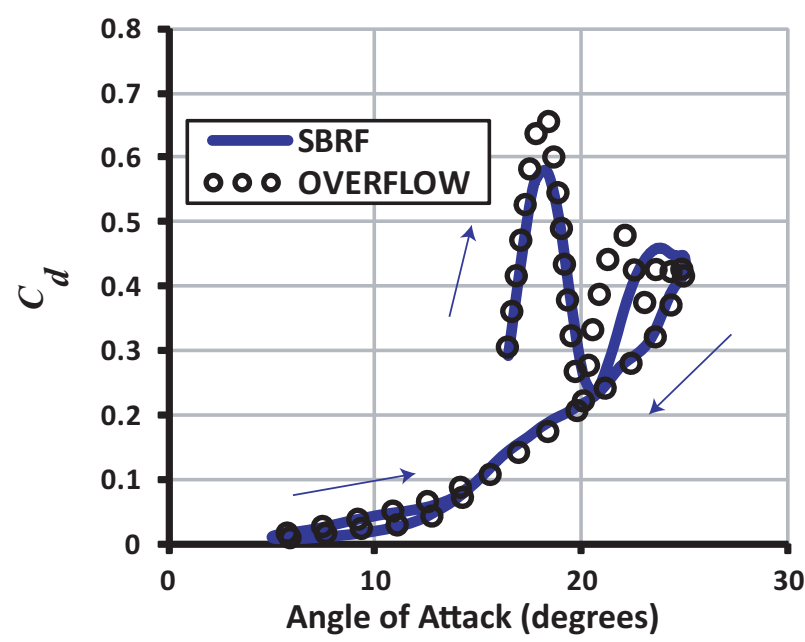

(a) $\theta_{G}=15^{\circ}, \bar{\theta}=10^{\circ}(2.6 \%$ error $)$

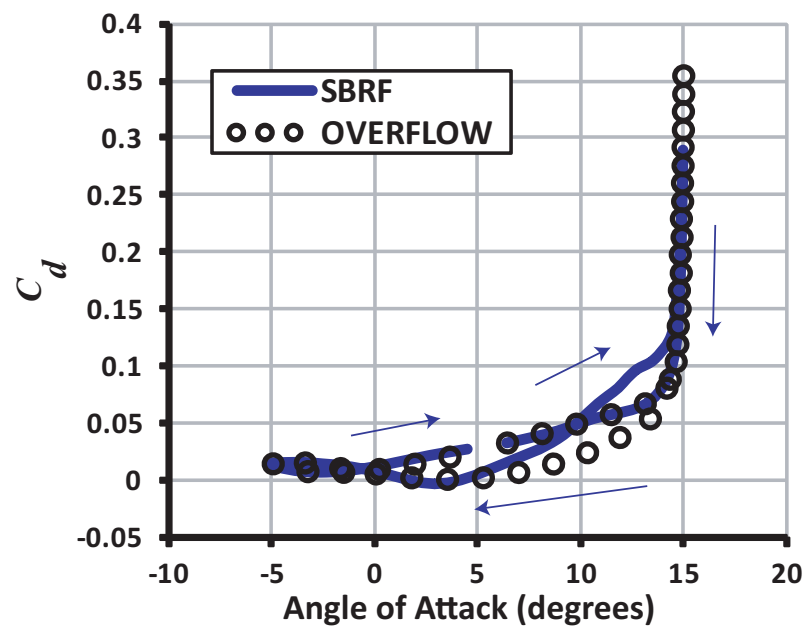

(c) $\theta_{G}=5^{\circ}, \bar{\theta}=10^{\circ}(2.9 \%$ error $)$

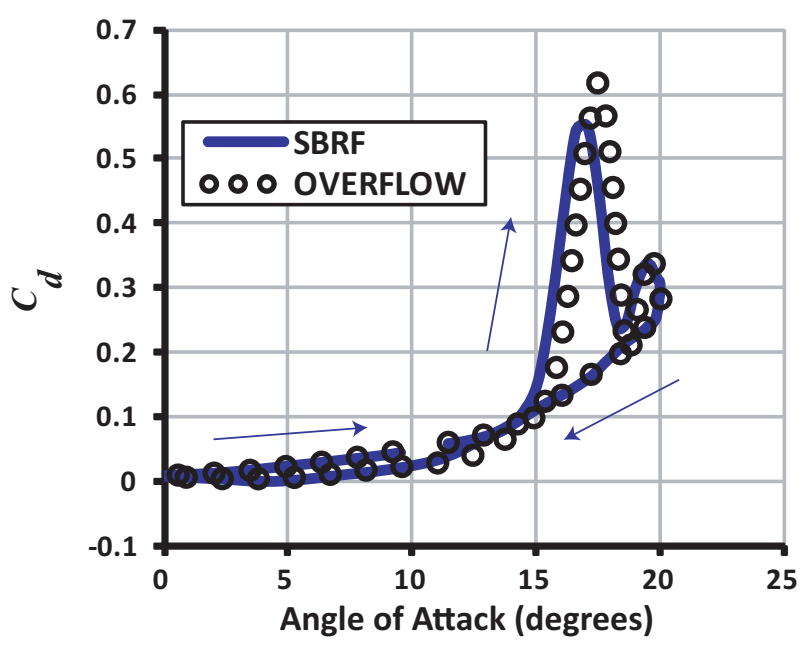

(b) $\theta_{G}=10^{\circ}, \bar{\theta}=10^{\circ}(2.4 \%$ error $)$

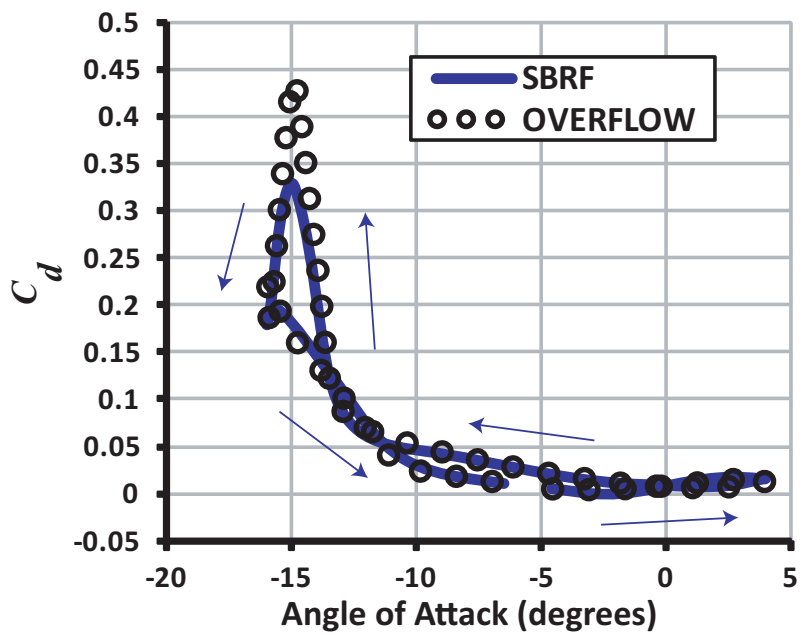

(d) $\theta_{G}=-6^{\circ}, \bar{\theta}=10^{\circ}(1.9 \%$ error $)$

Figure 8. Unsteady drag coefficients under dynamic stall conditions for a pitching airfoil $M=0.3, k_{\theta}=0.05$.

$\bar{h} / b=2 \%, k_{h}=0.088$, and $M_{0}=0.48$. Figure 10 (a),(c), and (e) correspond to $\bar{M}=0.1$, while (b), (d), and (f) correspond to $\bar{M}=0.22$. The level of agreement evident in Fig. 10 indicates that the implementation in OVERFLOW for combined airfoil oscillations under time-varying Mach number conditions is correct.

\section{IV.B.2. Dynamic Stall due to Realistic Airfoil Motion}

The results in this section demonstrate the effectiveness of the SBRF approach for modeling dynamic stall effects corresponding to airfoil motions representative of helicopter rotor blade dynamics. The accuracy of the SBRF predictions was quantified by comparing with OVERFLOW results for a set of test cases that were not included in the OLH used to train the surrogate mapping function. The full-order CFD data was generated for 2 rotor blade revolutions - i.e. 2 periods corresponding to $\omega_{1}$ - so that initial transients in the solutions were eliminated. The data from the final period was used for training and testing the SBRF. In order to reduce computer memory requirements for constructing the surrogates, the number of sample points was reduced by setting $\Delta t=8 \Delta t_{\mathrm{CFD}}$, where $\Delta t_{\mathrm{CFD}}$ is the time-step associated with the CFD solutions. This sampling rate resulted in a discretization of 88 time-steps per rotor revolution, which was found to be sufficient for capturing high frequency content in the aerodynamic responses. Note that the reduced-order $\mathrm{SBRF}$ predictions based on $\Delta t=8 \Delta t_{\mathrm{CFD}}$ are validated against the unfiltered full-order signals corresponding to $\Delta t_{\mathrm{CFD}}$ in this study.

Since the spanwise blade station is fixed at $r=0.75$ for all cases considered in this study, 7 variables are 


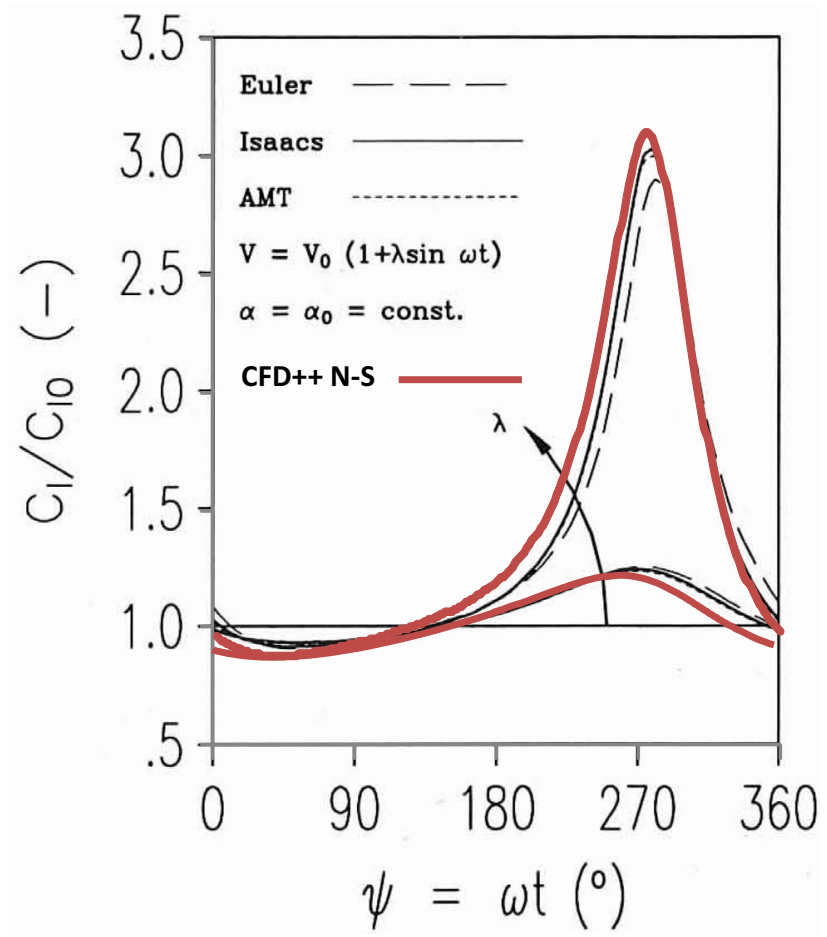

Figure 9. Comparison of unsteady lift predictions by CFD++ with results presented in Ref. 58 for time-varying Mach numbers $(\lambda=0.4,0.8)$

required to define a training case: $\left(\theta_{G}, \theta_{1}, \bar{\theta}, k_{\theta}, \bar{h}, k_{h}, \mu\right)$. Two OLH's were considered for training purposes; the first is valid for $12^{\circ} \leq \theta_{G} \leq 16^{\circ}$ and the second corresponds to $8^{\circ} \leq \theta_{G}<12^{\circ}$. Therefore, one surrogate was used for predictions corresponding to $12^{\circ} \leq \theta_{G} \leq 16^{\circ}$, while the other was used for $8^{\circ} \leq \theta_{G}<12^{\circ}$. Each OLH consisted of 125 cases, resulting in 250 total training cases. The bounds on the other OLH parameters were:

$$
\begin{gathered}
2^{\circ} \leq \theta_{1} \leq 15^{\circ} \\
0^{\circ} \leq \bar{\theta} \leq 4.5^{\circ} \\
0 \leq k_{\theta} \leq 0.3(8.2 / \mathrm{rev}) \\
0 \% \leq \bar{h} / b \leq 65 \% \\
0 \leq k_{h} \leq 0.15(4.1 / \mathrm{rev}) \\
0.3 \leq \mu \leq 0.4
\end{gathered}
$$

The upper bound for $\bar{\theta}$ was selected according to the 1st torsional mode shape of an MBB B0-105 blade calculated with the geometrically nonlinear beam model described in Ref. 59. A maximum torsional deflection of $5^{\circ}$ at the blade tip corresponds to approximately $4.5^{\circ}$ at the $75 \%$ spanwise station according to the 1 st torsional mode shape. Typical rotor blades are not likely to exceed such values for elastic deformation since larger deflections would correspond to high blade stresses. The upper bounds for $k_{\theta}$ and $k_{h}$ were chosen to represent the first two torsional and bending modal frequencies of a hingeless blade. The upper bound for $\bar{h}$ corresponds to a maximum effective angle of attack $\alpha_{\text {eff }}=12^{\circ}$, where $\alpha_{\text {eff }}$ is given by Eq. (38). The bounds 


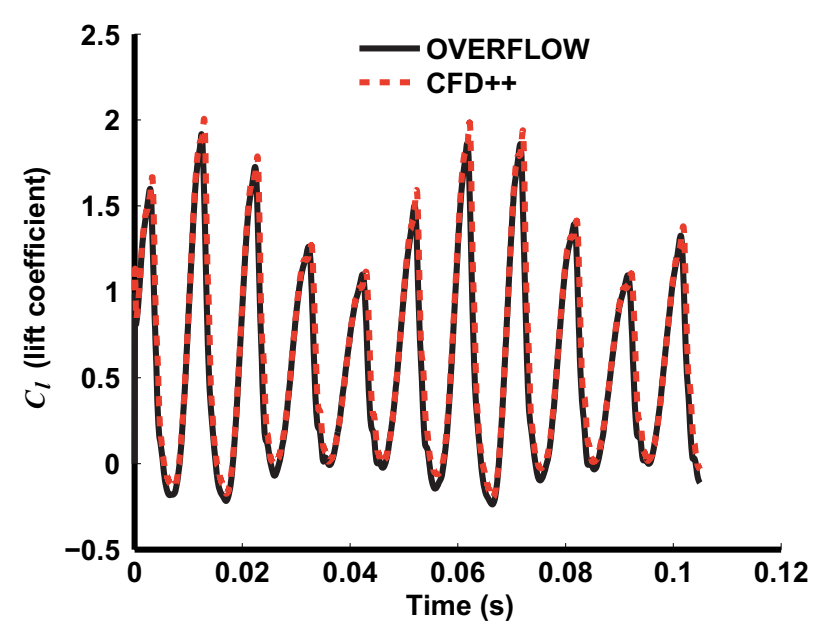

(a) Lift, $\bar{M}=0.1$

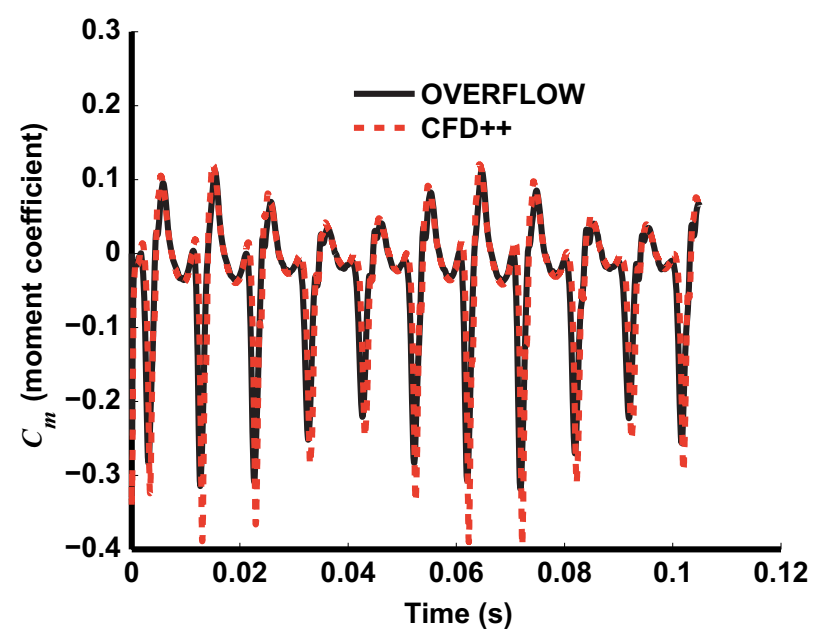

(c) Moment, $\bar{M}=0.1$

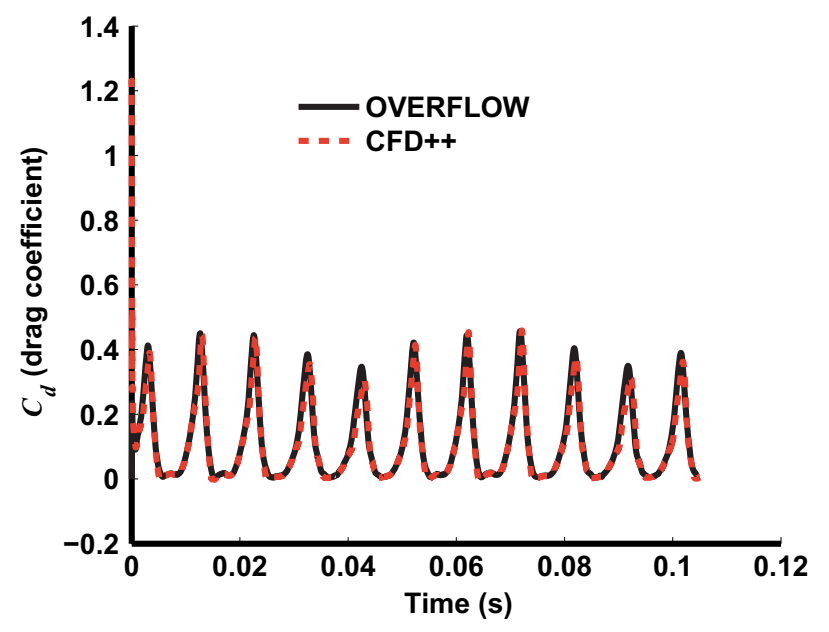

(e) Drag, $\bar{M}=0.1$

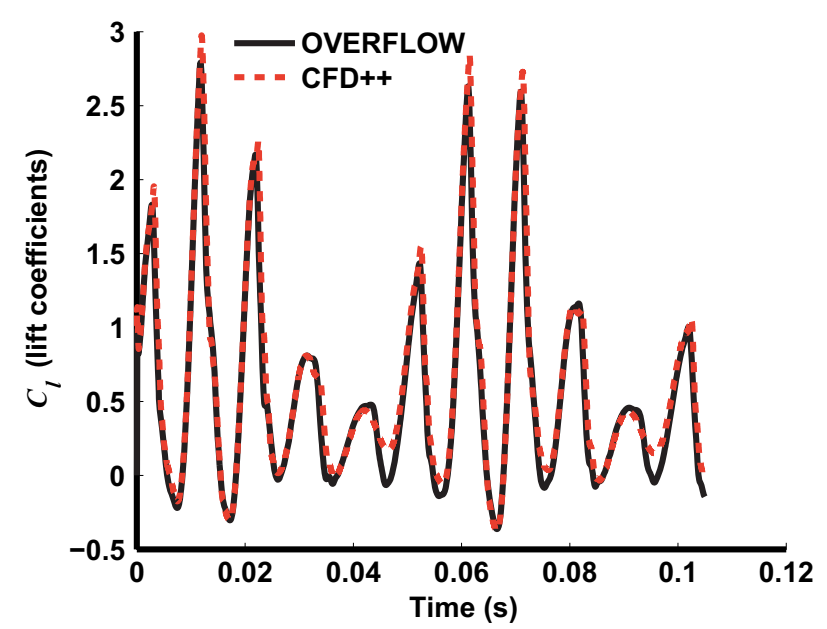

(b) Lift, $\bar{M}=0.22$

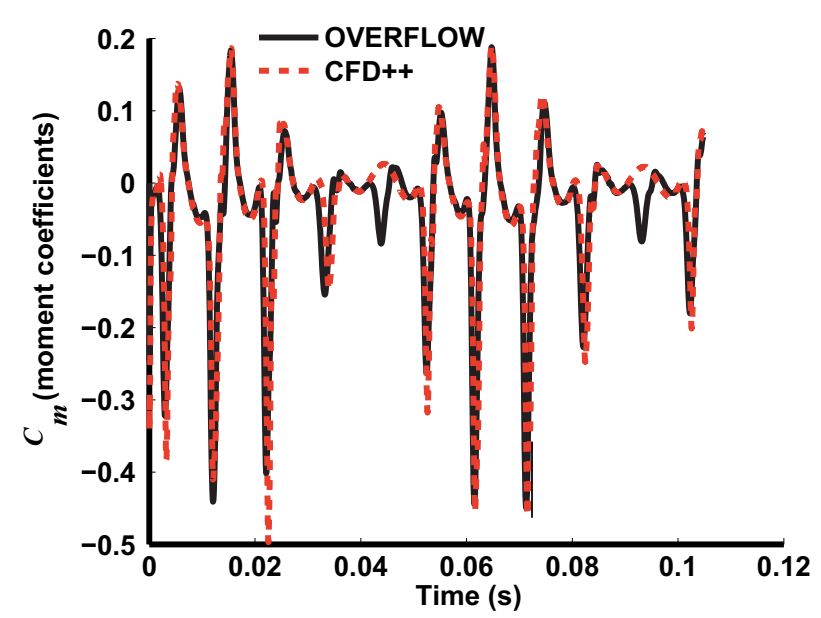

(d) Moment, $\bar{M}=0.22$

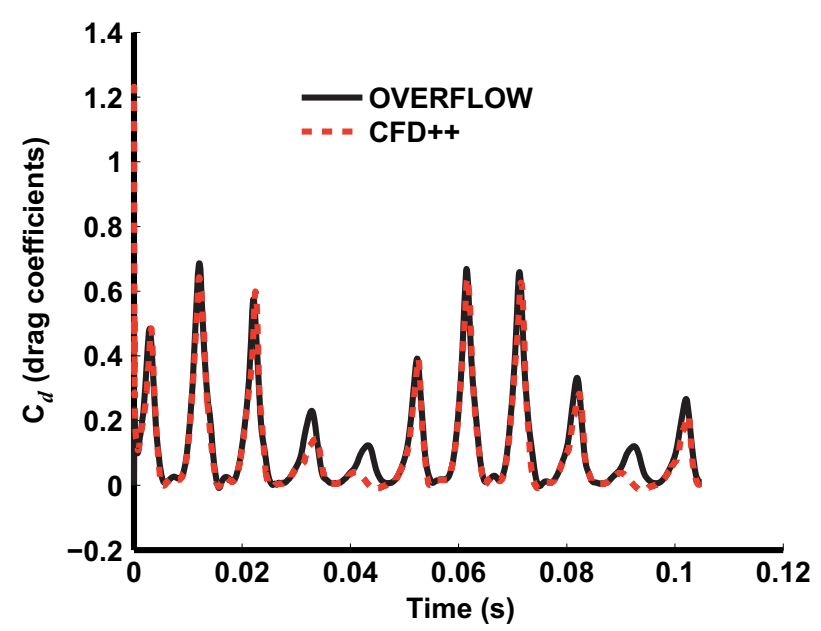

(f) Drag, $\bar{M}=0.22$

Figure 10. Comparison between OVERFLOW and CFD++ for combined motion cases. 
Table 3. SBRF errors for 9 test cases corresponding to pitch/plunge oscillations and time-varying freestream Mach numbers

\begin{tabular}{ccc}
\hline \hline Error in $\boldsymbol{C}_{\boldsymbol{l}}$ & Error in $\boldsymbol{C}_{\boldsymbol{m}}$ & Error in $\boldsymbol{C}_{\boldsymbol{d}}$ \\
\hline $1.5-3.7 \%$ (avg. $=2.5 \%)$ & $1.7-9.7 \%$ (avg. $=4.6 \%)$ & $1.7-6.6 \%$ (avg. $=3.6 \%)$ \\
\hline \hline
\end{tabular}

on the advance ratio $\mu$ represent high speed forward flight conditions for which dynamic stall may occur.

$$
\alpha_{\mathrm{eff}}(t)=\theta(t)+\frac{\dot{h}(t)}{V(t)}, \quad V(t)=a M(t) .
$$

The SBRF prediction errors (Eq. (31)) corresponding to 9 test cases that were not inlcuded in the training data set are provided in Table 3. The average errors over all 9 test cases were $2.5 \%, 4.6 \%$, and $3.9 \%$ for lift, moment, and drag respectively. These results demonstrate that overall, the SBRF prediction errors are very low relative to the peak-to-peak values of the responses.

Representative hysteresis loops for 6 of the 9 test cases are provided in Figs. 11 - 13. As with the simplified motion cases considered in Section IV.A, the SBRF accurately models the nonlinear hystertic behavior associated with the combined oscillations. In addition, the test cases include both advancing(e.g. Fig. 12(b)) and retreating (e.g. Fig. 12(f)) side moment stall. Therefore, the cases considered in this study provided an excellent test of the SBRF approach since both mechanisms of helicopter rotor blade stall were captured. It should be noted that the SBRF approach required a fraction of a second on a single computer processor to generate reduced-order predictions, while the full-order OVERFLOW solutions required $7-12$ hours on 3 processors. Therefore, the SBRF exhibits the computational efficiency required for a variety of aeroelasticity and design studies.

It is clear from Table 3 and Figs. 11 - 13 that the SBRF reduced-order modeling approach is capable of accurately approximating full-order dynamic stall solutions. In order to provide context regarding the maximum SBRF errors, i.e. the worst case scenarios, the SBRF, OVERFLOW, and CFD++ predictions are compared in Fig. 14 for the 2 test cases corresponding to the highest errors in the SBRF moment coefficient predictions. The unsteady aerodynamic responses are plotted versus the azimuth angle, $\omega_{1} t$. The case depicted in Fig. 14(a) - (c) corresponds to the one shown in Fig 12(a), in which a noticeable SBRF overprediction of the moment stall was observed. The second case corresponds to the maximum error in the moment coefficient (9.7\% in Table 3) and is shown in Fig. 14(d) - (f). From a visual inspection of Fig. 14, it is clear that the SBRF predictions are much closer to the OVERFLOW solutions than the CFD++ predictions. Therefore, the worst case SBRF predictions are as accurate as those from another CFD solver. Such a comparison could be used to guide the decision process regarding whether the SBRF is sufficiently accurate or whether additional training cases should be generated. For example, it could be concluded that the SBRF based on 250 training cases considered in this study is sufficiently accurate since the maximum error cases fall within the bounds of uncertainty associated with different CFD codes.

\section{Conclusions}

The surrogate based recurrence framework (SBRF) approach to reduced-order nonlinear unsteady aerodynamic modeling was shown to effectively mimic full-order flow solutions under dynamic stall conditions. Results pertaining to combined pitch/plunge airfoil oscillations under time-varying Mach number conditions demonstrate that the SBRF approach can accurately model unsteady airloads associated with realistic helicopter rotor blade dynamics. Once constructed from a limited number of full-order CFD analyses, the SBRF predictions require a fraction of a second to compute, while maintaining a level of fidelity corresponding to CFD results that would require several hours to obtain. Since the SBRF retains the fidelity associated with CFD solutions of dynamic stall effects, while exhibiting the computational efficiency of semi-empirical models, it is ideally suited for a variety of rotary-wing aeroelasticity and active/passive design optimization studies. The principal results of this study are:

1. The effectiveness of the SBRF approach for modeling the nonlinear hysteretic behavior associated with dynamic stall was demonstrated by considering airfoils simultaneously oscillating in pitch and plunge, while subjected to time-varying freestream Mach numbers representative of a helicopter rotor 


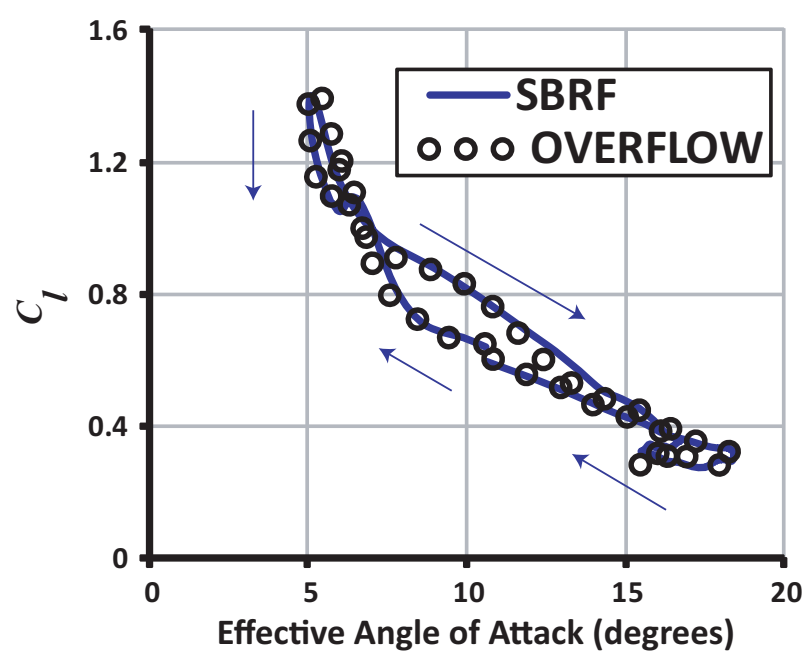

(a) $\theta_{G}=11.1^{\circ}, \theta_{1}=6.9^{\circ}, \bar{\theta}=0.5^{\circ}, k_{\theta}=0.01, \bar{h} / b=22 \%$, $k_{h}=0.11, \mu=0.33(2.8 \%$ error $)$

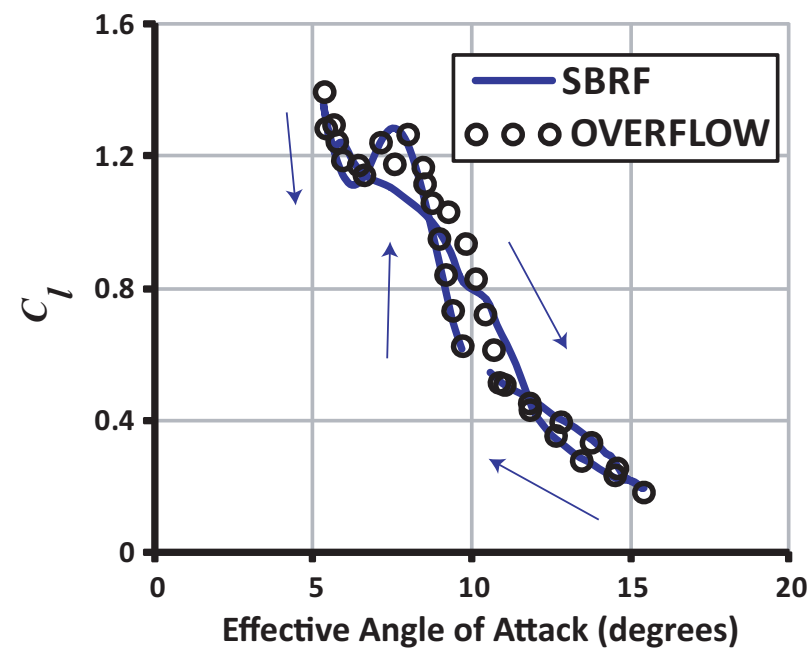

(c) $\theta_{G}=10.3^{\circ}, \theta_{1}=4.8^{\circ}, \bar{\theta}=0.18^{\circ}, k_{\theta}=0.18, \bar{h} / b=32 \%$, $k_{h}=0.01, \mu=0.36$ (2.4\% error)

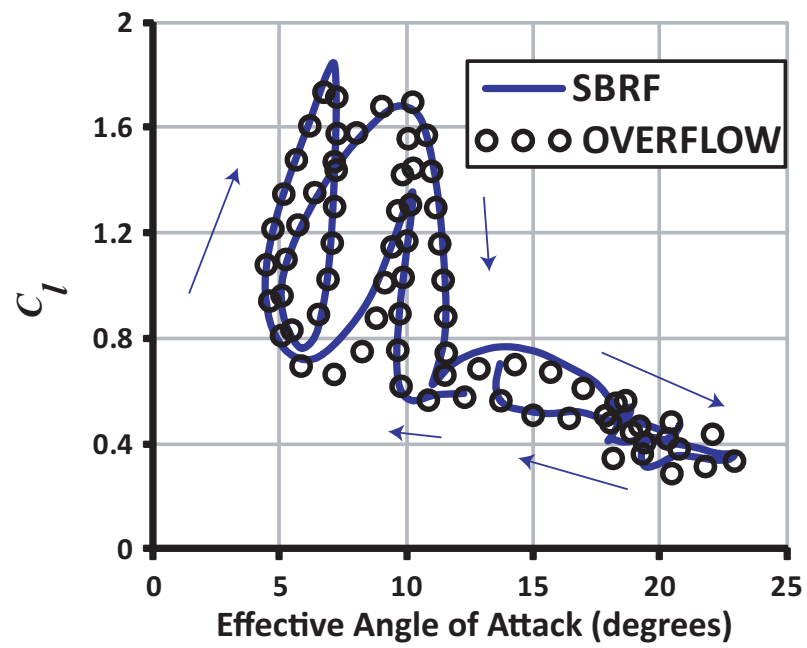

(e) $\theta_{G}=13.4^{\circ}, \theta_{1}=7.8^{\circ}, \bar{\theta}=1.6^{\circ}, k_{\theta}=0.24, \bar{h} / b=5 \%$, $k_{h}=0.04, \mu=0.32(2.8 \%$ error $)$

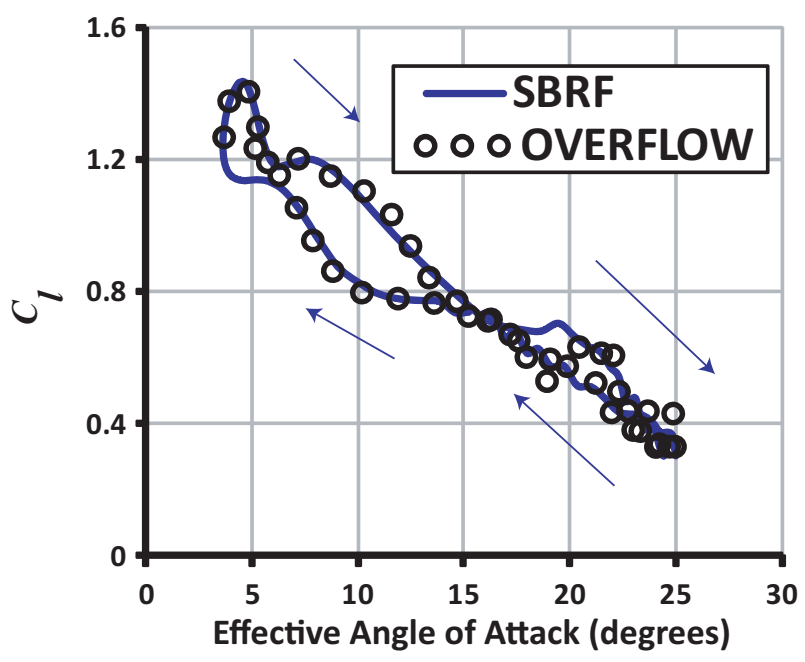

(b) $\theta_{G}=15^{\circ}, \theta_{1}=10.7^{\circ}, \bar{\theta}=0.7^{\circ}, k_{\theta}=0.15, \bar{h} / b=16 \%$, $k_{h}=0.07, \mu=0.37$ ( $2.7 \%$ error $)$

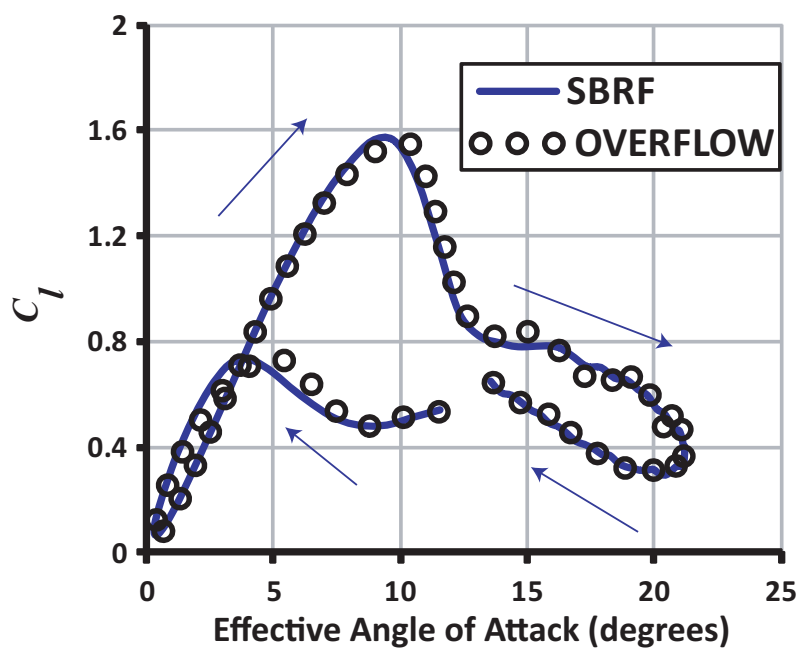

(d) $\theta_{G}=12.6^{\circ}, \theta_{1}=10.3^{\circ}, \bar{\theta}=3.1^{\circ}, k_{\theta}=0.06, \bar{h} / b=36 \%$, $k_{h}=0.02, \mu=0.34$ ( $1.8 \%$ error)

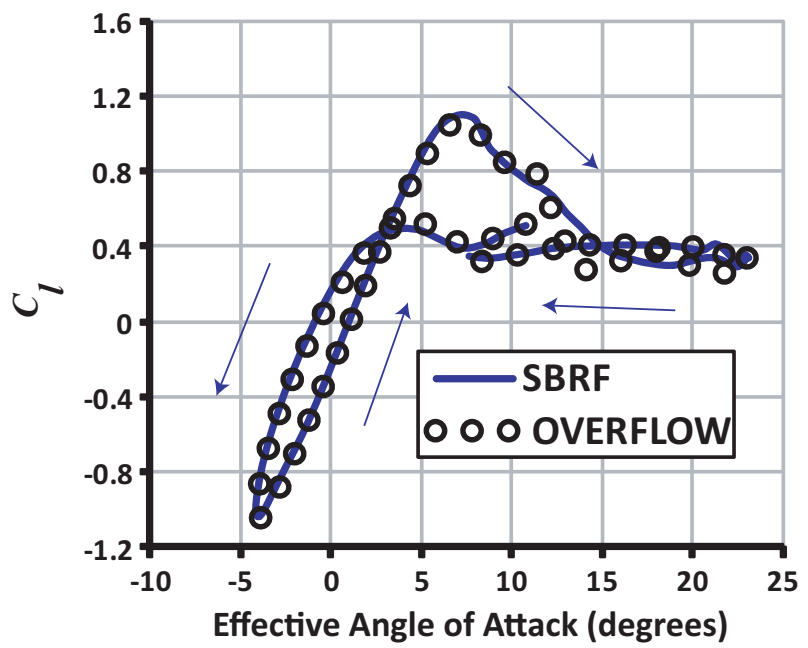

(f) $\theta_{G}=9.5^{\circ}, \theta_{1}=11.6^{\circ}, \bar{\theta}=2.7^{\circ}, k_{\theta}=0.08, \bar{h} / b=3 \%$, $k_{h}=0.13, \mu=0.36$ (1.5\% error)

Figure 11. Unsteady lift coefficients under dynamic stall conditions. 


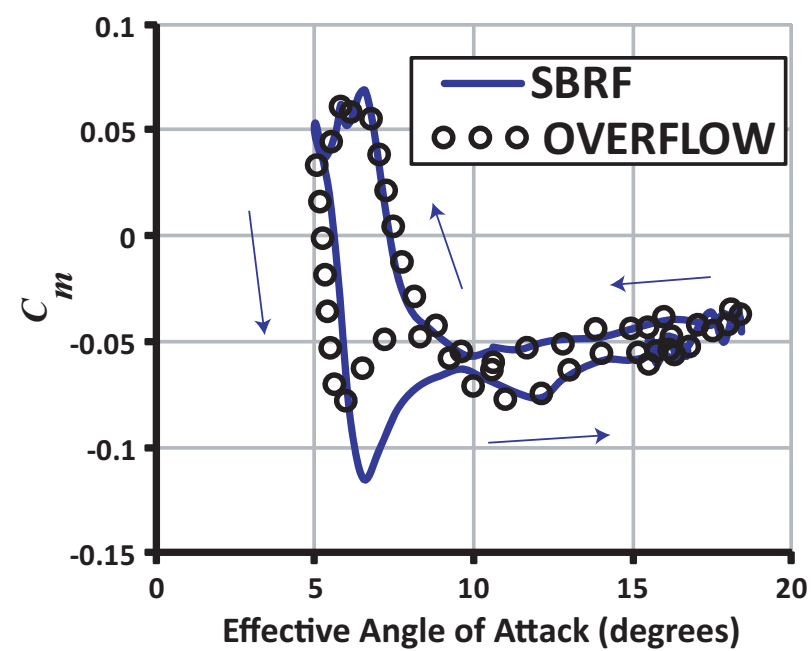

(a) $\theta_{G}=11.1^{\circ}, \theta_{1}=6.9^{\circ}, \bar{\theta}=0.5^{\circ}, k_{\theta}=0.01, \bar{h} / b=22 \%$, $k_{h}=0.11, \mu=0.33$ (6.0\% error)

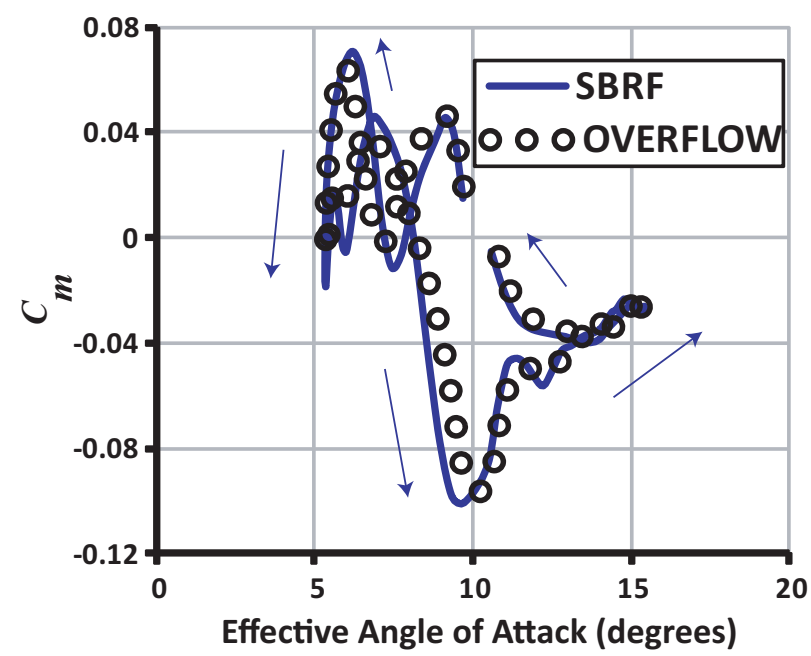

(c) $\theta_{G}=10.3^{\circ}, \theta_{1}=4.8^{\circ}, \bar{\theta}=0.18^{\circ}, k_{\theta}=0.18, \bar{h} / b=32 \%$, $k_{h}=0.01, \mu=0.36$ ( $4.7 \%$ error $)$

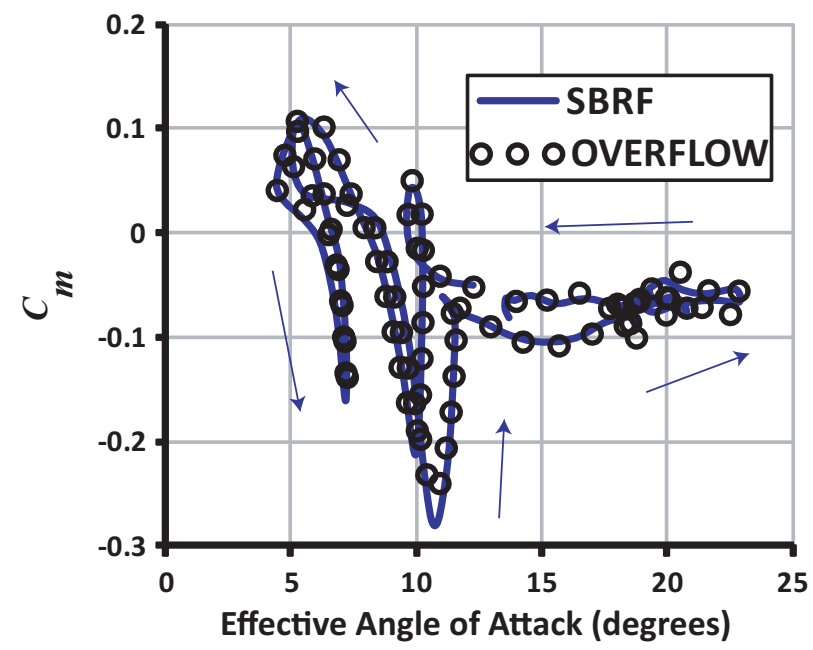

(e) $\theta_{G}=13.4^{\circ}, \theta_{1}=7.8^{\circ}, \bar{\theta}=1.6^{\circ}, k_{\theta}=0.24, \bar{h} / b=5 \%$, $k_{h}=0.04, \mu=0.32$ (2.7\% error)

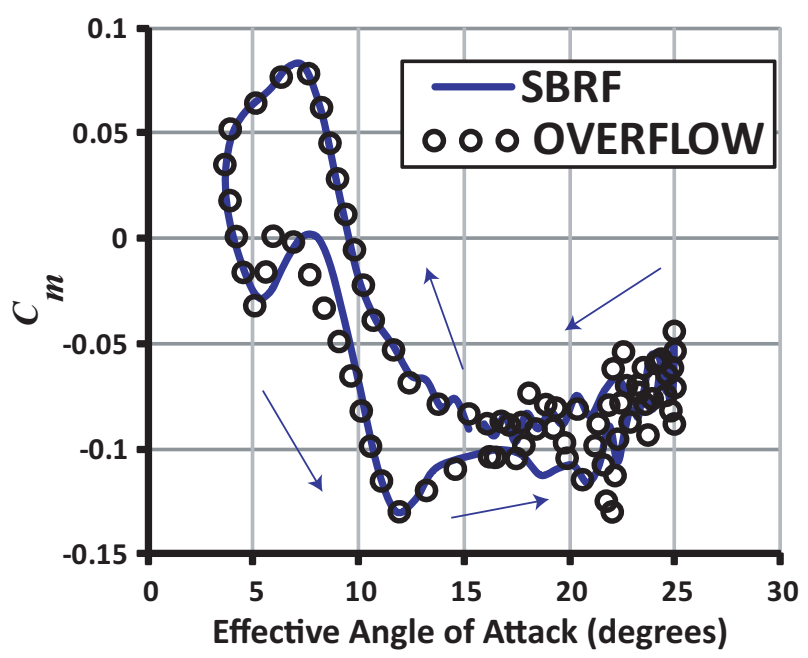

(b) $\theta_{G}=15^{\circ}, \theta_{1}=10.7^{\circ}, \bar{\theta}=0.7^{\circ}, k_{\theta}=0.15, \bar{h} / b=16 \%$, $k_{h}=0.07, \mu=0.37$ (4.6\% error)

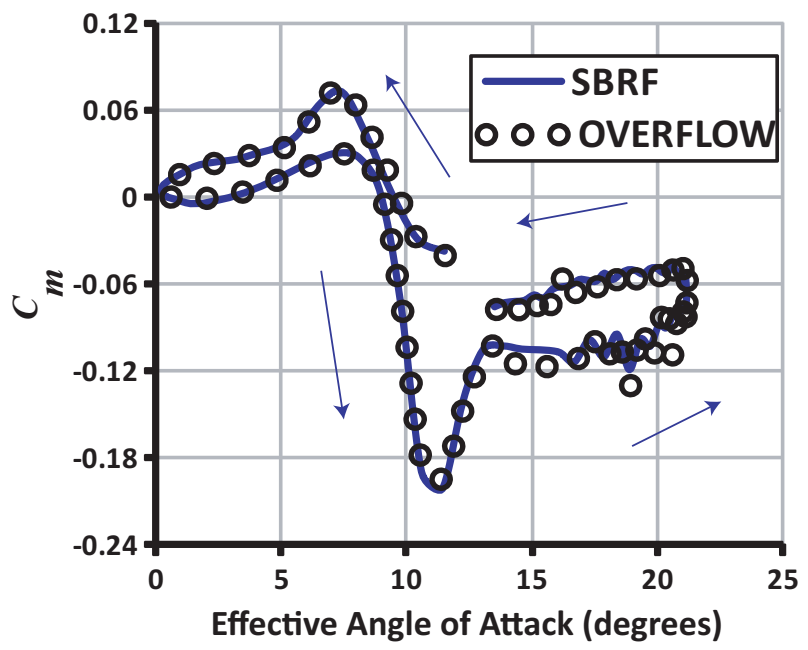

(d) $\theta_{G}=12.6^{\circ}, \theta_{1}=10.3^{\circ}, \bar{\theta}=3.1^{\circ}, k_{\theta}=0.06, \bar{h} / b=36 \%$, $k_{h}=0.02, \mu=0.34$ (1.7\% error)

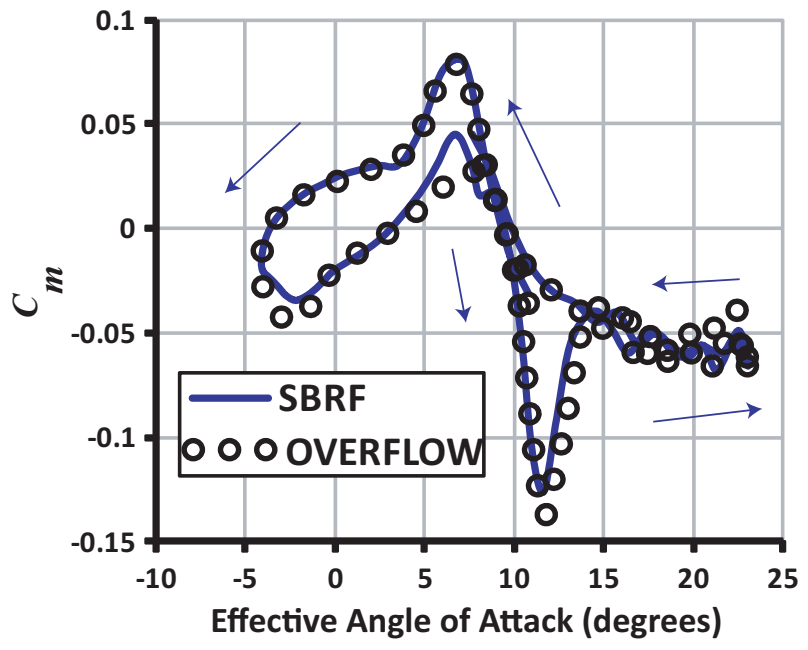

(f) $\theta_{G}=9.5^{\circ}, \theta_{1}=11.6^{\circ}, \bar{\theta}=2.7^{\circ}, k_{\theta}=0.08, \bar{h} / b=3 \%$, $k_{h}=0.13, \mu=0.36$ (2.7\% error)

Figure 12. Unsteady moment coefficients under dynamic stall conditions. 


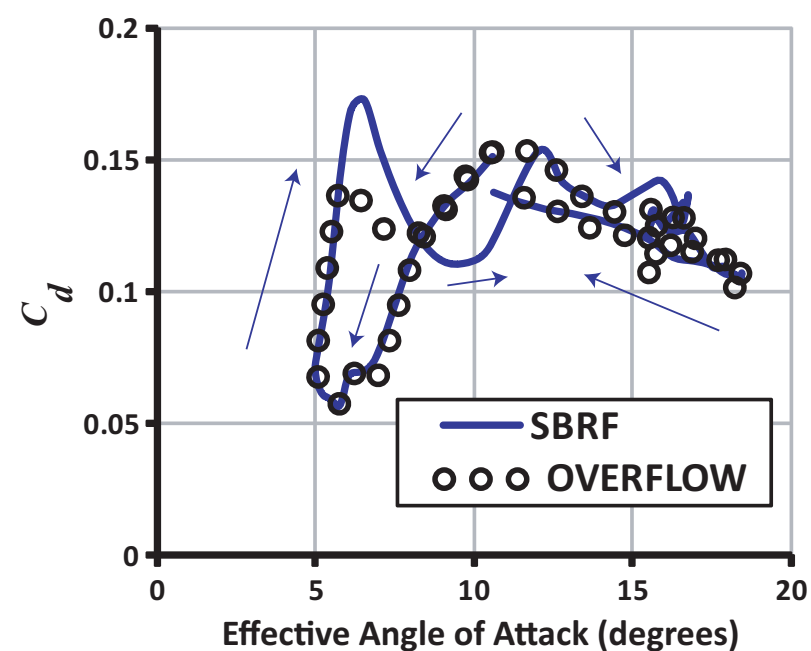

(a) $\theta_{G}=11.1^{\circ}, \theta_{1}=6.9^{\circ}, \bar{\theta}=0.5^{\circ}, k_{\theta}=0.01, \bar{h} / b=22 \%$, $k_{h}=0.11, \mu=0.33$ (6.6\% error)

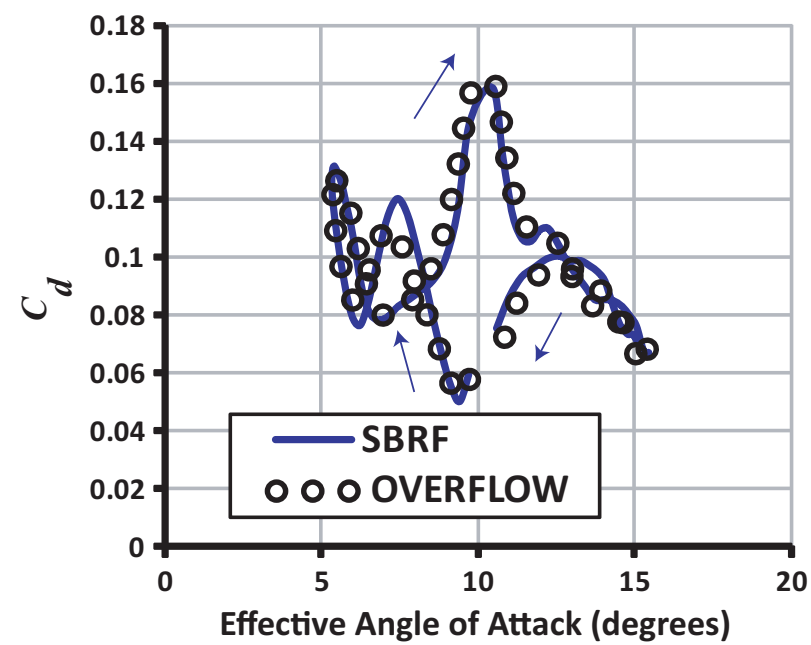

(c) $\theta_{G}=10.3^{\circ}, \theta_{1}=4.8^{\circ}, \bar{\theta}=0.18^{\circ}, k_{\theta}=0.18, \bar{h} / b=32 \%$, $k_{h}=0.01, \mu=0.36$ (4.1\% error)

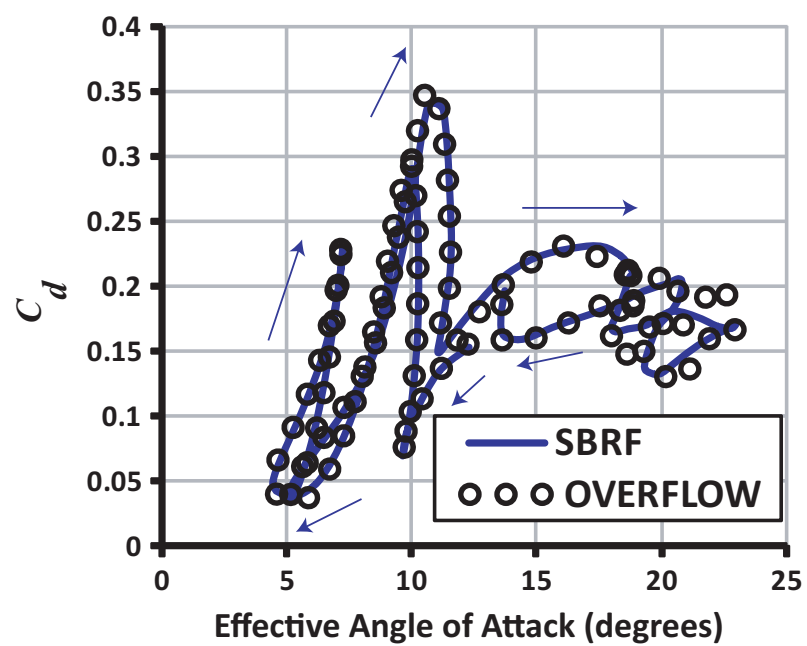

(e) $\theta_{G}=13.4^{\circ}, \theta_{1}=7.8^{\circ}, \bar{\theta}=1.6^{\circ}, k_{\theta}=0.24, \bar{h} / b=5 \%$ $k_{h}=0.04, \mu=0.32$ (2.1\% error)

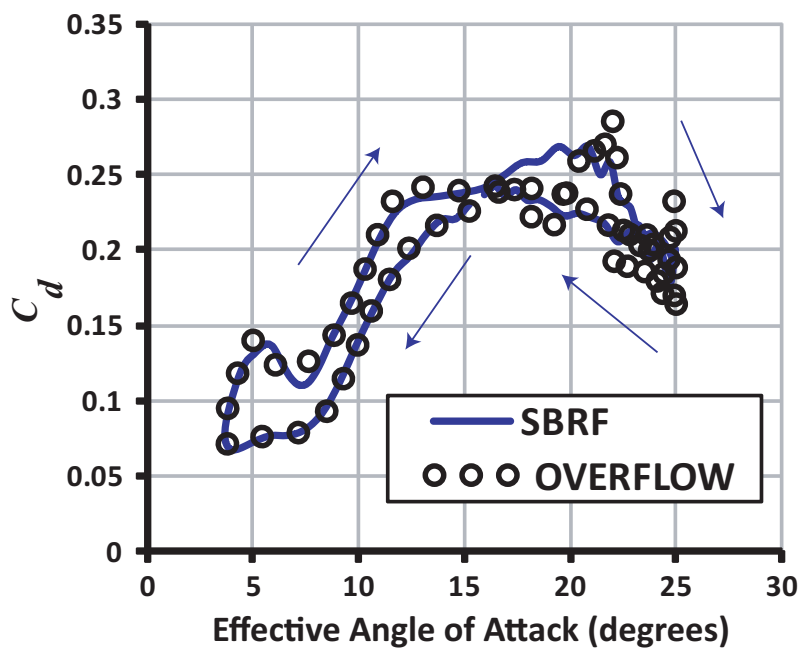

(b) $\theta_{G}=15^{\circ}, \theta_{1}=10.7^{\circ}, \bar{\theta}=0.7^{\circ}, k_{\theta}=0.15, \bar{h} / b=16 \%$, $k_{h}=0.07, \mu=0.37$ ( $4.4 \%$ error)

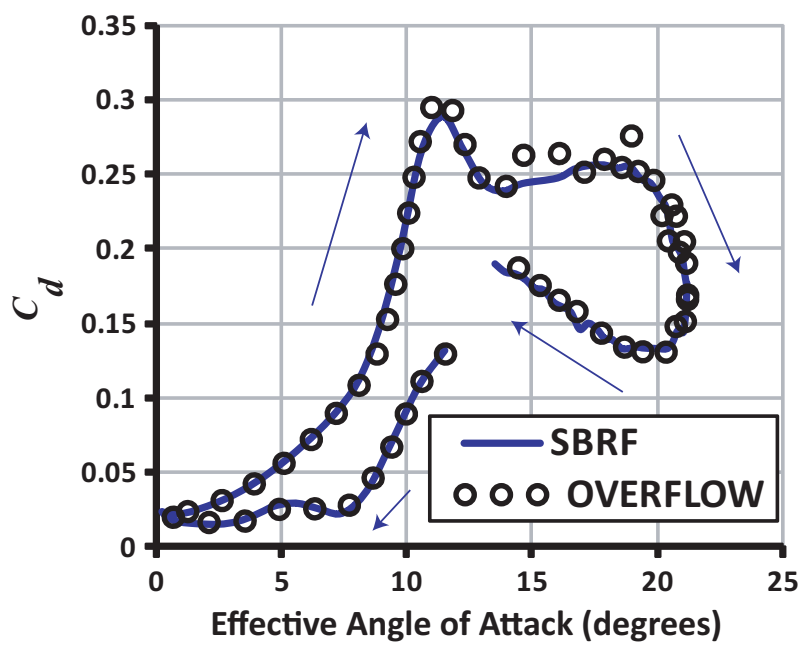

(d) $\theta_{G}=12.6^{\circ}, \theta_{1}=10.3^{\circ}, \bar{\theta}=3.1^{\circ}, k_{\theta}=0.06, \bar{h} / b=36 \%$, $k_{h}=0.02, \mu=0.34$ (1.7\% error)

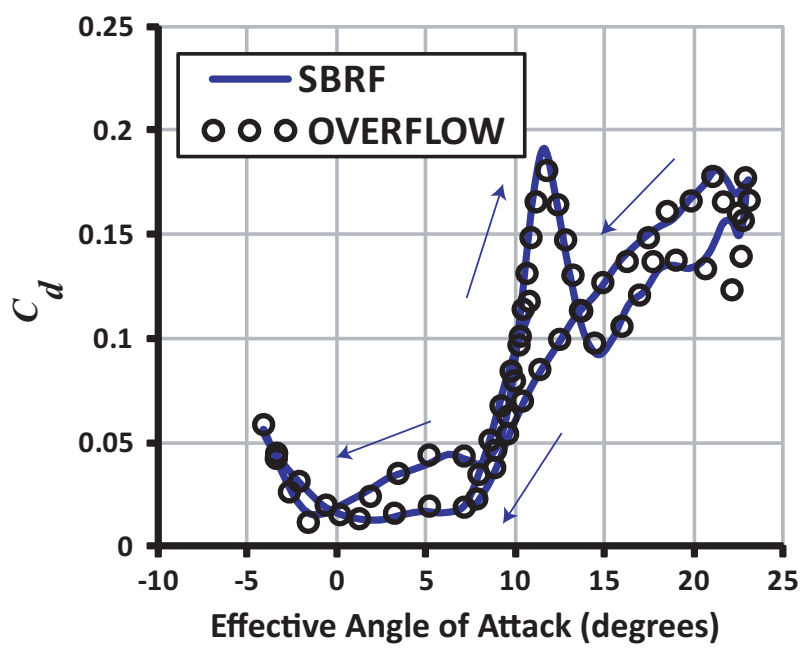

(f) $\theta_{G}=9.5^{\circ}, \theta_{1}=11.6^{\circ}, \bar{\theta}=2.7^{\circ}, k_{\theta}=0.08, \bar{h} / b=3 \%$, $k_{h}=0.13, \mu=0.36$ (2.6\% error)

Figure 13. Unsteady drag coefficients under dynamic stall conditions. 


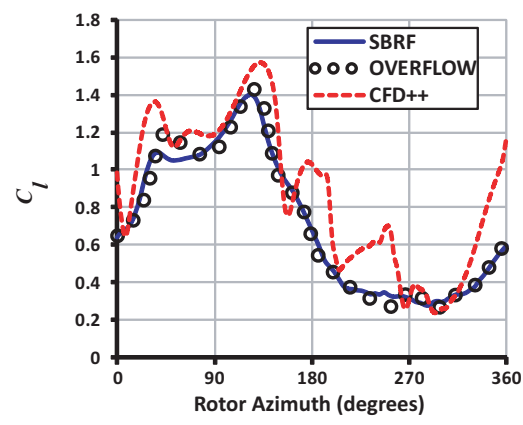

(a) Lift Coefficient $\left(\theta_{G}=11.1^{\circ}, \theta_{1}=\right.$ $6.9^{\circ}, \bar{\theta}=0.5^{\circ}, k_{\theta}=0.01, \bar{h} / b=22 \%$, $\left.k_{h}=0.11, \mu=0.33\right)$

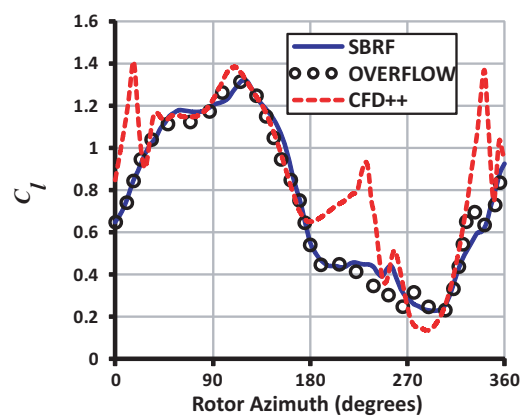

(d) Lift Coefficient $\left(\theta_{G}=14.2^{\circ}, \theta_{1}=\right.$ $5.3^{\circ}, \bar{\theta}=1.8^{\circ}, k_{\theta}=0.12, \bar{h} / b=47 \%$, $\left.k_{h}=0.13, \mu=0.35\right)$

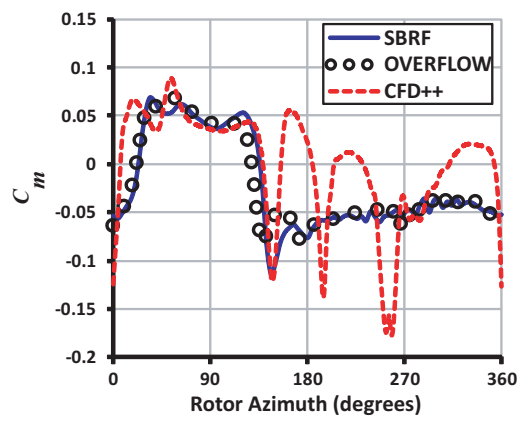

(b) Moment Coefficient $\left(\theta_{G}=11.1^{\circ}\right.$, $\theta_{1}=6.9^{\circ}, \bar{\theta}=0.5^{\circ}, k_{\theta}=0.01, \bar{h} / b=$ $\left.22 \%, k_{h}=0.11, \mu=0.33\right)$

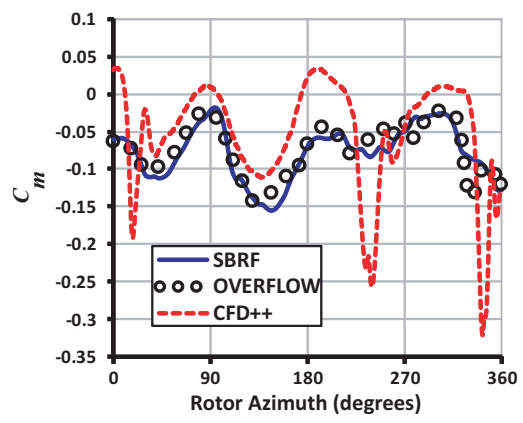

(e) Moment Coefficient $\left(\theta_{G}=14.2^{\circ}\right.$, $\theta_{1}=5.3^{\circ}, \bar{\theta}=1.8^{\circ}, k_{\theta}=0.12, \bar{h} / b=$ $47 \%, k_{h}=0.13, \mu=0.35$ )

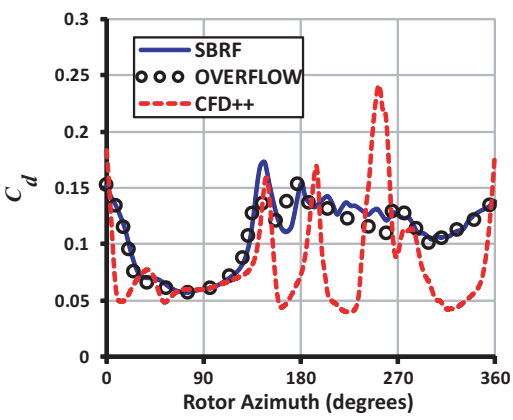

(c) Drag Coefficient $\left(\theta_{G}=11.1^{\circ}, \theta_{1}=\right.$ $6.9^{\circ}, \bar{\theta}=0.5^{\circ}, k_{\theta}=0.01, \bar{h} / b=22 \%$, $\left.k_{h}=0.11, \mu=0.33\right)$

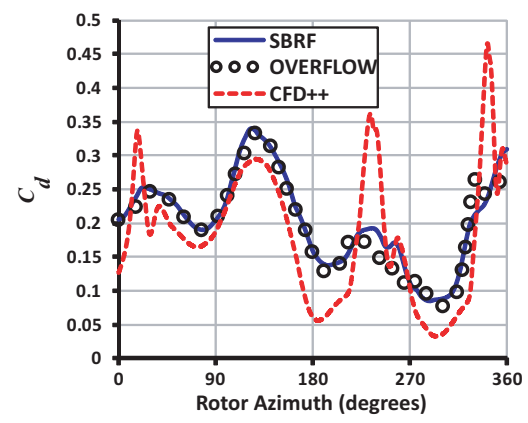

(f) Drag Coefficient $\left(\theta_{G}=14.2^{\circ}, \theta_{1}=\right.$ $5.3^{\circ}, \bar{\theta}=1.8^{\circ}, k_{\theta}=0.12, \bar{h} / b=47 \%$, $\left.k_{h}=0.13, \mu=0.35\right)$

Figure 14. Comparison of SBRF, OVERFLOW, and CFD++.

in forward flight.

2. In addition to accounting for realistic blade dynamics, the SBRF approach accurately modeled fullorder solutions corresponding to shock induced separation and excessive angles of attack; thus the SBRF approach can effectively model advancing and retreating blade stall associated with helicopter rotors.

3. Even in the maximum error cases, the SBRF predictions were sufficiently accurate so as to fall well within the uncertainty bounds associated with different CFD solvers.

\section{Acknowledgments}

This research was supported in part by an NSF sponsored AGEP postdoctoral fellowship for B. Glaz. Partial support from the Center for Rotorcraft Innovation under WBS 2009-B-11-05.3-A17, as well as the Vertical Lift Research Center of Excellence (VLRCOE) sponsored by the NRTC with Dr. M. Rutkowski as grant monitor is also hereby acknowledged.

\section{References}

16.

${ }^{1}$ Carr, L. W., "Progress in Analysis and Prediction of Dynamic Stall," Journal of Aircraft, Vol. 25, No. 1, 1988, pp. 6 -

${ }^{2}$ Leishman, J. G., Principles of Helicopter Aerodynamics, Cambridge University Press, Second ed., 2006.

${ }^{3}$ Carta, F. O., "An Analysis of the Stall Flutter Instability of Helicopter Rotor Blades," Journal of the American Helicopter Society, Vol. 12, No. 4, 1967, pp. $1-18$.

${ }^{4}$ Johnson, W., Helicopter Theory, Dover, 1980.

${ }^{5}$ Petot, D., "Differential Equation Modeling of Dynamic Stall," La Recherche Aérospatiale, Vol. 5, 1989, pp. 59-71.

${ }^{6}$ Petot, D., Arnaoud, G., Harrison, R., Stevens, J., Dieterich, O., van der Wall, B. G., Young, C., and Szechenyi, E., "Stall 
Effects and Blade Torsion - An Evaluation of Predictive Tools," Journal of the American Helicopter Society, Vol. 44, No. 4, 1999, pp. $320-331$.

${ }^{7}$ Leishman, J. G. and Beddoes, T. S., "A Generalized Model for Unsteady Aerodynamic Behavior and Dynamic Stall Using the Indicial Method," Journal of the American Helicopter Society, Vol. 34, No. 3, 1989, pp. 3 - 17.

${ }^{8}$ Datta, A., Nixon, M., and Chopra, I., "Review of Rotor Loads Prediction with the Emergence of Rotorcraft CFD," Journal of the American Helicopter Society, Vol. 52, No. 4, Oct. 2007, pp. $287-317$.

${ }^{9}$ Strawn, R. C., Caradonna, F. X., and Duque, P. N., "30 Years of Rotorcraft Computational Fluid Dynamics Research and Development," Journal of the American Helicopter Society, Vol. 51, No. 1, 2006, pp. 5 - 21.

${ }^{10}$ Bhagwat, M. J. and Ormiston, R. A., "Examination of Rotor Aerodynamics in Steady and Maneuvering Flight Using CFD and Conventional Methods," Proceedings of the American Helicopter Society Aeromechanics Specialists Conference, San Francisco, CA, January 2008.

${ }^{11}$ Bain, J., Sankar, L. N., Prasad, J. V. R., Bauchau, O. A., Peters, D. A., and He, C., "Computational Modeling of Variable-Droop Leading Edge in Forward Flight," Journal of Aircraft, Vol. 46, No. 2, 2009, pp. 617 - 626.

${ }^{12}$ Chandrasekhara, M. S., Martin, P. B., and Tung, C., "Compressible Dynamic Stall Performance of a Variable Droop Leading Edge Airfoil with a Gurney Flap," Journal of the American Helicopter Society, Vol. 53, No. 1, 2008, pp. 18 - 25.

${ }^{13}$ Liu, L., Friedmann, P. P., Kim, I., and Bernstein, D. S., "Rotor Performance Enhancement and Vibration Reduction in Presence of Dynamic Stall Using Actively Controlled Flaps," Journal of the American Helicopter Society, Vol. 53, No. 4, 2008, pp. $338-350$.

${ }^{14}$ Hassan, A. A. and JanakiRam, R. D., "Effects of Zero-Mass Synthetic Jets on the Aerodynamics of the NACA-0012 Airfoil," Journal of the American Helicopter Society, Vol. 43, No. 4, 1998, pp. 303 - 311.

${ }^{15}$ Lucia, D. J., Beran, P. S., and Silva, W. A., "Reduced-order Modeling: New Approaches for Computational Physics," Progress in Aerospace Sciences, Vol. 40, 2004, pp. 51 - 117.

${ }^{16}$ Hall, K. C., Thomas, J. P., and Dowell, E. H., "Proper Orthogonal Decomposition Technique for Transonic Unsteady Aerodynamic Flows," AIAA Journal, Vol. 38, No. 10, Oct. 2000, pp. 1853 - 1862.

${ }^{17}$ Silva, W., "Identification of Nonlinear Aeroelastic Systems Based on the Volterra Theory: Progress and Opportunities," Nonlinear Dynamics, Vol. 39, 2005, pp. 25 - 62.

${ }^{18}$ Balajewicz, M., Nitzsche, F., and Feszty, D., "Application of Multi-Input Volterra Theory to Nonlinear Multi-Degree-ofFreedom Aerodynamic Systems," AIAA Journal, Vol. 48, No. 1, 2010, pp. $56-62$.

${ }^{19}$ Liu, L., Friedmann, P. P., and Padthe, A. K., "An Approximate Unsteady Aerodynamic Model for Flapped Airfoils Including Improved Drag Predictions," Proceedings of the 34th European Rotorcraft Forum, Liverpool, UK, Sept. 2008.

${ }^{20}$ Trizila, P. C., Kang, C., Visbal, M. R., and Shyy, W., "A Surrogate Model Approach in 2D Versus 3D Flapping Wing Aerodynamic Analysis," 12th AIAA/ISSMO Multidisciplinary Analysis and Optimization Conference, Victoria, British Columbia, Sept. 2008, AIAA Paper 2008-5914

${ }^{21}$ Suresh, S., Omkar, S. N., Mani, V., and Guru Prakash, T. N., "Lift Coefficient Prediction at High Angle of Attack Using Recurrent Neural Network," Aerospace Science and Technology, Vol. 7, No. 1, 2003, pp. 595 - 602.

${ }^{22}$ Marques, F. D. and Anderson, J., "Identification and Prediction of Unsteady Transonic Aerodynamic Loads by MultiLayer Functionals," Journal of Fluids and Structures, Vol. 15, 2001, pp. 83 - 106.

${ }^{23}$ Faller, W. E. and Schreck, S. J., "Unsteady Fluid Mechanics Applications of Neural Networks," Journal of Aircraft, Vol. 34, No. 1, 1997, pp. $48-55$.

${ }^{24}$ Glaz, B., Liu, L., and Friedmann, P. P., "Reduced Order Nonlinear Unsteady Aerodynamic Modeling Using a Surrogate Based Approach," Proceedings of the American Helicopter Society Aeromechanics Specialists Conference, San Francisco, CA, January 2010.

${ }^{25}$ Glaz, B., Liu, L., and Friedmann, P. P., "Reduced Order Nonlinear Unsteady Aerodynamic Modeling Using a Surrogate Based Recurrence Framework," AIAA Journal, 2010, submitted for publication.

${ }^{26}$ Simpson, T. W., Peplinski, D., Koch, P. N., and Allen, J. K., "Metamodels for Computer-based Engineering Design: Survey and recommendations," Engineering with Computers, Vol. 17, 2001, pp. 129-150.

${ }^{27}$ Queipo, N. V., Haftka, R. T., Shyy, W., Goel, T., Vaidyanathan, R., and Tucker, P. K., "Surrogate-Based Analysis and Optimization," Progress in Aerospace Sciences, Vol. 41, 2005, pp. 1-28.

${ }^{28}$ Forrester, A., Sóbester, A., and Keane, A., Engineering Design via Surrogate Modelling: A Practical Guide, AIAA, 2008.

${ }^{29}$ Leontaritis, I. J. and Billings, S. A., "Input-Output Parametric Models for Nonlinear Systems," Int. Journal of Control, Vol. 41, No. 2, 1985, pp. $303-344$.

${ }^{30}$ Levin, A. U. and Narendra, K. S., "Control of Nonlinear Dynamical Systems Using Neural Networks - Part II: Observability, Identification, and Control," IEEE Transactions on Neural Networks, Vol. 7, No. 1, January 1996, pp. 30 - 42.

${ }^{31}$ Xiang, C., "Existence of Global Input-output Model for Nonlinear Systems," Proceedings of the International Conference on Control and Automation, Budapest, Hungary, June 2005.

${ }^{32}$ Ljung, L., System Identification: Theory for the User, Prentice Hall, Upper Saddle River, NJ, 1999.

${ }^{33}$ Bisplinghoff, R. L., Ashley, H., and Halfman, R., Aeroelasticity, Addison-Wesley, 1955, pg. 272.

${ }^{34}$ Jose, A. I., Leishman, J. G., and Baeder, J. D., "Unsteady Aerodynamic Modeling with Time-Varying Free-Stream Mach Numbers," Journal of the American Helicopter Society, Vol. 51, No. 4, Oct. 2006, pp. 299 - 318.

${ }^{35}$ Mandic, D. P. and Chambers, J. A., Recurrent Neural Networks for Prediction, John Wiley \& Sons, New York, USA, 2001.

${ }^{36}$ Pearson, R. K., "Nonlinear Input/Output Modelling," Journal of Process Control, Vol. 5, No. 4, 1995, pp. 197 - 211.

${ }^{37}$ Simpson, T. W., Peplinski, D., Koch, P. N., and Allen, J. K., "Sampling Strategies for Computer Experiments: Design and Analysis," Int. Journal of Reliability and Applications, Vol. 2, No. 3, 2001, pp. 209 - 240.

${ }^{38}$ Sacks, J., Welch, W. J., Mitchell, T. J., and Wynn, H. P., "Design and Analysis of Computer Experiments," Statistical Science, Vol. 4, No. 4, 1989, pp. 409-435. 
${ }^{39}$ Martin, J. and Simpson, T., "Use of Kriging Models to Approximate Deterministic Computer Models," AIAA Journal, Vol. 43, No. 4, April 2005, pp. 853-863.

${ }^{40}$ Simpson, T. W., Booker, A. J., Ghosh, D., Giunta, A. A., Koch, P. N., and Yang, R., "Approximation Methods in Multidisciplinary Analysis and Optimization: A Panel Discussion," Structural and Multidisciplinary Optimization, Vol. 27, No. 5, July 2004, pp. 302-313.

${ }^{41}$ McKay, M. D., Beckman, R. J., and Conover, W. J., "A Comparison of Three Methods for Selecting Values of Input Variables in the Analysis of Output from a Computer Code," Technometrics, Vol. 21, No. 2, May 1979, pp. $239-245$.

42 Jin, R., Chen, W., and Sudjianto, A., "An Efficient Algorithm for Constructing Optimal Design of Computer Experiments," Journal of Statistical Planning and Inference, Vol. 134, No. 1, 2005, pp. 268 - 287.

${ }^{43}$ Koch, P. N., Evans, J. P., and Powell, D., "Interdigitation for Effective Design Space Exploration using iSIGHT," Structural and Multidisciplinary Optimization, Vol. 23, No. 2, 2002, pp. 111-126.

${ }^{44}$ Jones, D. R., "A Taxonomy of Global Optimization Methods Based on Response Surfaces," Journal of Global Optimization, Vol. 21, 2001, pp. $345-383$.

${ }^{45}$ Lophaven, S. N., Nielsen, H. B., and Søndergaard, J., "A Matlab Kriging Toolbox, Version 2.0," Informatics and Mathematical Modeling, DTU, 2002, Technical Report IMM-TR-2002-12.

${ }^{46}$ Jones, D. R., "The DIRECT global optimization algorithm," Encyclopedia of Optimization, Vol. 1, 2001, pp. 431-440.

${ }^{47}$ Sasena, M., Flexibility and Efficiency Enhancements for Constrained Global Optimization with Kriging Approximations, Ph.D. thesis, University of Michigan, 2002, Mechanical Engineering.

${ }^{48}$ Duque, E. P. N., Sankar, L. N., Menon, S., Bauchau, O., Ruffin, S., Smith, M., Ahuja, A., Brentner, K. S., Long, L. N., Morris, P. J., and Gandhi, F., "Revolutionary Physics-Based Design Tools for Quiet Helicopters," Proceedings of 44th AIAA Aerospace Sciences Meeting and Exhibit, Reno, NV, January 2006, AIAA Paper 2006-1068.

${ }^{49}$ Peroomian, O., Chakravarthy, S., Palaniswamy, S., and Goldberg, U., "Convergence Acceleration for Unified-Grid Formulation Using Preconditioned Implicit Relaxation," AIAA Paper 98-0116, Reno, NV, January 1998.

${ }^{50}$ Peroomian, O., Chakravarthy, S., and Goldberg, U., "A "Grid-Transparent" Methodology for CFD," AIAA Paper 970724, Reno, NV, January 1997.

${ }^{51}$ Vassberg, J., Tinoco, E., Mani, M., Brodersen, O., Eisfeld, B., Wahls, R., Morrison, J., Zickuhr, T., Laflin, K., and Mavriplis, D., "Summary of the Third AIAA CFD Drag Prediction Workshop," Proceedings of 45th AIAA Aerospace Sciences Meeting and Exhibit, Reno, NV, January 2007, AIAA Paper 2007-260.

${ }^{52}$ Potsdam, M., Yeo, H., and Johnson, W., "Rotor Airloads Prediction using Loose Aerodynamic/Structural Coupling," Proceedings of the 60th American Helicopter Society Annual Forum, Baltimore, MD, June 2004.

${ }^{53}$ Schoenenberg, M., Dyakonov, A., Buning, P., Scallion, B., and Van Norman, J., "Aerodyanmic Challenges for the Mars Science Laboratory Entry Descent and Landing," Proceedings of the 41st AIAA Thermophysics Conference, San Antonio, TX, June 2009, AIAA Paper 2009-3914.

${ }^{54}$ Fang, Y. and Menon, S., "A Two-Equation Subgrid Model for Large-Eddy Simulation of High Reynolds Number Flows," Proceedings of 44th AIAA Aerospace Sciences Meeting and Exhibit, Reno, NV, January 2006, AIAA Paper $2006-0116$.

${ }^{55}$ Chan, W., "The OVERGRID Interface for Computational Simulations on Overset Grids," Proceedings of the 32nd AIAA

Fluid Dynamics Conference and Exhibit, St, Louis, MO, June 2002, AIAA Paper 2002-3188.

${ }^{56}$ Nichols, R. and Buning, P., "Users Manual for OVERFLOW 2.1," August 2008.

${ }^{57}$ Chakravarthy, S. R. and Osher, S., "New Class of High Accuracy TVD Schemes for Hyperbolic Conservation Laws," Proceedings of the 23rd AIAA Aerospace Sciences Meeting and Exhibit, Reno, NV, Jan 1985, AIAA Paper No. 85-0363.

${ }^{58}$ van der Wall, B. G. and Leishman, J. G., "On the Influence of Time-Varying Flow Velocity on Unsteady Aerodynamics," Journal of the American Helicopter Society, Vol. 39, No. 4, Oct. 1994.

${ }^{59}$ Yuan, K. A. and Friedmann, P. P., Aeroelastic and Structural Optimization of Composite Helicopter Rotor Blades with Swept Tips, NASA CR 4665, June 1995. 